\title{
Super-Resolution Reconstruction of Remote Sensing Images Using Multiple-Point Statistics and Isometric Mapping
}

\author{
Ting Zhang ${ }^{1}$ (D), Yi Du ${ }^{2}$ and Fangfang $L u^{1, *}$ \\ 1 College of Computer Science and Technology, Shanghai University of Electric Power, 2588 Changyang Road, \\ Shanghai 200090, China; tingzh@shiep.edu.cn \\ 2 School of Engineering, Shanghai Polytechnic University, 2360 Jinhai Road, Shanghai 201209, China; \\ duyi@sspu.edu.cn \\ * Correspondence: lufangfang@shiep.edu.cn; Tel.: +86-189-1703-8329
}

Academic Editors: Gonzalo Pajares Martinsanz, Lizhe Wang, Sangram Ganguly and Richard Gloaguen Received: 13 April 2017; Accepted: 10 July 2017; Published: 15 July 2017

\begin{abstract}
When using coarse-resolution remote sensing images, super-resolution reconstruction is widely desired, and can be realized by reproducing the intrinsic features from a set of coarse-resolution fraction data to fine-resolution remote sensing images that are consistent with the coarse fraction information. Prior models of spatial structures that encode the expected features at the fine (target) resolution are helpful to constrain the spatial patterns of remote sensing images to be generated at that resolution. These prior models can be used properly by multiple-point statistics (MPS), capable of extracting the intrinsic features of patterns from prior models such as training images, and copying them to the simulated regions using hard and soft conditional data, or even without any conditional data. However, because traditional MPS methods based on linear dimensionality reduction are not suitable to deal with nonlinear data, and isometric mapping (ISOMAP) can reduce the dimensionality of nonlinear data effectively, this paper presents a sequential simulation framework for generating super-resolution remote sensing images using ISOMAP and MPS. Using four different examples, it is demonstrated that the structural characteristics of super-resolution reconstruction of remote sensing images using this method, are similar to those of training images.
\end{abstract}

Keywords: remote sensing images; dimensionality reduction; super-resolution reconstruction; soft data; training image

\section{Introduction}

Remote sensing images obtained by remote sensing imaging systems, are widely used in understanding and management in landscape ecology, natural resource planning, and environmental systems. Super-resolution remote sensing can be considered as an inverse problem that reconstructs a high-resolution (or fine-resolution) image from a set of coarse-resolution class fractions [1,2]. However, due to the physical limitation of relevant imaging devices in remote sensing, scaling up ground and remotely sensed data to the desired spatial resolutions, is unavoidable [3]. For super-resolution reconstruction of continua in remotely sensed images, Atkinson et al. [4] studied the downscaling cokriging, which provides an incremental development for the use of cokriging for image fusion described in Ref. [5]. Boucher and Kyriakidis [1] proved that many approaches for remote sensing using super-resolution adopted prior models of spatial structure, either implicitly or explicitly (see for example Ref. [6]). Boucher et al. [2] also proposed a geostatistical solution for super-resolution of remote sensing images using sequential indicator simulation to obtain multiple possible stochastic results. Pan et al. [7] proposed a super-resolution reconstruction method of remote sensing images 
based on compressive sensing, structural self-similarity, and dictionary learning. Generally speaking, super-resolution reconstruction is a process of combining one or multiple low-resolution spatial images to produce a high-resolution image, and is a powerful tool to acquire the desired spatial resolution in remote sensing.

Various super-resolution methods have been proposed, which can be categorized into three classes [8-10]: interpolation-based methods [11-13], multi-image-based methods [14-16], and example learning-based methods [17-20]. The interpolation-based methods apply a base function or an interpolation kernel to predict the pixels in high-resolution grids. However, they only perform well in smooth (low-frequency) areas, but poorly in edge (high-frequency) areas. The multi-image-based methods recover a high-resolution image by fusing a set of low-resolution images of the same scene, but they are restricted by the difficulties of acquiring enough low-resolution images.

The last category of super-resolution methods is based on example learning, assuming that the lost information in a low-resolution image can be learned from a set of training images [21]. Training images are numerical prior models containing the structures and relationships existing in realistic models. The example learning-based methods acquire a high-resolution image by inferring the mapping relationship between the high-resolution and low-resolution image pairs, so a set of high-resolution training examples should be prepared in advance for learning. Obviously, this kind of method heavily relies on the quality of the training image sets.

To address the super-resolution issue based on example learning for remote sensing, one can use a prior model to limit the under-determination caused by missing or incomplete spatial information. Once the prior model of spatial structures has been determined, it provides a probability distribution of corresponding variables studied in above super-resolution issue. Actually, the probability distribution hidden in the prior model contains the uncertainty about the unknown attribute values to be reconstructed given the current available conditional information. Also, the uncertainty in probability distribution may lead to multiple results providing an assessment of uncertainty regarding remote sensing images, instead of generating only one single image from the conditional data [1,2].

As one of the main branches of stochastic simulation, and introduced by the seminal work of Guardiano and Srivastava [22], multiple-point statistics (MPS) is a relatively broad concept, allowing capture of intrinsic structures from a prior model existing in a training image, and then copying them to the simulated region by reproducing high-order statistics conditioned to some conditional spatial data. Hence, MPS can fully use the given prior information and conditional data simultaneously [23,24]. In MPS, there are two types of conditional data to limit the simulated results: hard data, and soft data. For example, in the characterization of oil wells, Figure $1 \mathrm{a}, \mathrm{b}$ is, respectively, hard data and soft data in the same region $(250 \times 250$ units). The white and black points in Figure 1a are shale wells, and sandstone wells, respectively, with Figure $1 \mathrm{~b}$ describing the probability of sandstone hidden in the whole region. Soft data are usually abundant and much easier to be achieved than hard data, so they especially should be used for reconstructing the unknown models for better precision.

In addition to conditional data, another issue challenging MPS is that it is normally quite CPU-intensive, due to its large computational burden. Hence, some classical MPS methods, like filter-based simulation (FILTERSIM) [23], and distance-based pattern simulation (DISPAT) [24], use dimensionality reduction to decrease the redundancy existing in high-dimensional data extracted from training images, hopefully reducing the burdens of computation. In FILTERSIM, the patterns in training images are characterized by some filters to acquire the filter-based score space. DISPAT introduces multi-dimensional scaling (MDS) for dimensionality reduction of training images. Although MDS in DISPAT and filters used in FILTERSIM can reduce the dimensionality and help to discover the linear structures of training images existing in high-dimensional space, they fail to reconstruct nonlinear structures, because they essentially are linear dimensionality reduction methods. 


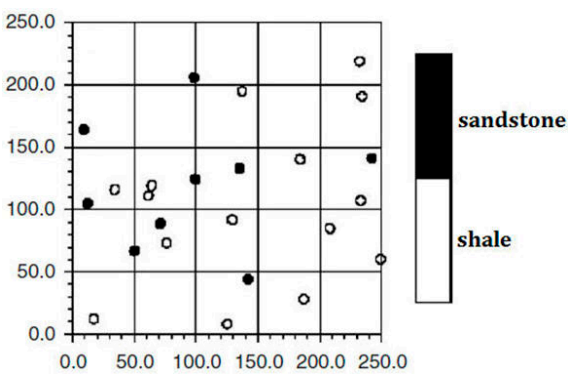

(a)

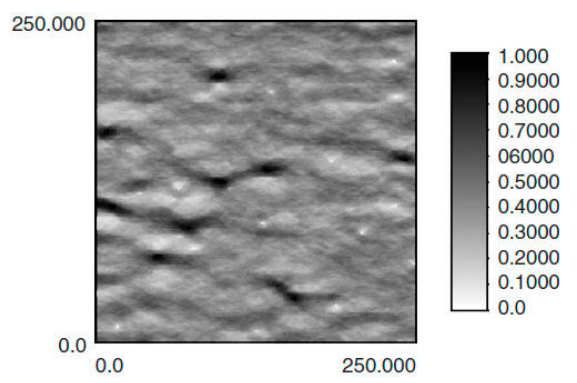

(b)

Figure 1. Sandstone wells and shale wells (hard data), and the probability image of sandstone (soft data). (a) Well data; (b) probability image of sandstone.

Normally, those structures with strong correlations in high-dimensional space can generate some results that lie on or close to a smooth low-dimensional manifold that is widely studied in differential geometry, as well as machine learning, known as "manifold learning" [25-28]. As a classical method in manifold learning for nonlinear dimensionality reduction, isometric mapping (ISOMAP) preserving geometry at all scales replaces Euclidean distances by geodesic distances, mapping nearby points on the manifold to nearby points and faraway points to faraway points in low-dimensional space [28].

The aim of this paper is to present an example learning-based method using MPS and ISOMAP for super-resolution of remote sensing images. Because of the capability brought by ISOMAP in nonlinear dimensionality reduction, ISOMAP is combined with MPS to be qualified for the functions as MDS in DISPAT, or filters in FILTERSIM. Besides, soft data are quite useful for improving reconstruction quality, so coarse fraction data from low-resolution images are used as soft data for reconstruction in our method. The remainder of this paper is organized as follows. Section 2 details the explanations for each step of the proposed method. Section 3 summarizes the main procedure of the method. Section 4 provides comparative experimental results, and Section 5 concludes this paper.

\section{Detailed Explanations for Each Step of the Proposed Method}

There are in total, 4 steps in the proposed method, which will be described in the following sections.

\subsection{Step 1: Building a Pattern Dataset from Training Images}

\subsubsection{Data Templates, Data Events and Patterns}

A training image is scanned with a data template $\tau_{D}$ that comprises a central location $\mathbf{u}$, and its $D$ neighboring locations $\mathbf{u}_{\alpha}(\alpha=1,2, \ldots, D)$. $\mathbf{u}_{\alpha}$ is defined as $\mathbf{u}_{\alpha}=\mathbf{u}+\mathbf{h}_{\alpha}$, where $\mathbf{h}_{\alpha}$ is the vector describing the data template. Considering that an attribute $S$ has $J$ possible states $\left\{s_{k} ; k=1,2, \ldots, J\right\}$, the data event $d\left(\mathbf{u}_{\alpha}\right)$ with the central location $\mathbf{u}$ is defined as [29]

$$
d\left(\mathbf{u}_{\alpha}\right)=\left\{S\left(\mathbf{u}_{\alpha}\right)=s_{k_{\alpha}} ; \alpha=1,2, \ldots, D . k_{\alpha} \in[1, J]\right\},
$$

where $S\left(\mathbf{u}_{\alpha}\right)$ is the state at the location $\mathbf{u}_{\alpha}$ within $\tau_{D}$, and $s_{k_{\alpha}}$ means one of the states of $s_{k}$. Figure 2a illustrates a data template with $9 \times 9$ nodes, in which the central node $\mathbf{u}$ is to be simulated. Figure $2 b, c$ are two data events captured by $\tau_{D}$. The different nodal colors in Figure $2 b, c$ mean different states of an attribute. Non-color square means the state of current node is unknown. Note that the term "node" here means a pixel in a 2D image. The data events extracted from training images are also called "patterns" representing the essential features of training images. For example, Figure 3 illustrates a pattern of a training image captured by a $3 \times 3$ data template. Only two states, the white node and the gray one, exist in the training image of Figure $3 \mathrm{~b}$. 


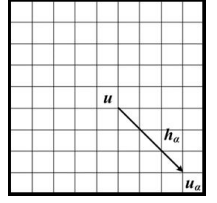

(a)

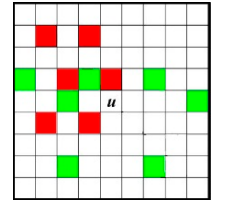

(b)

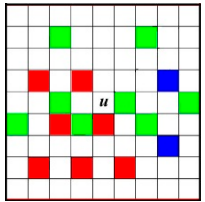

(c)

Figure 2. Illustrations of a $2 \mathrm{D}$ data template and two 2D data events. (a) A data template; (b) data event \#1; (c) data event \#2.

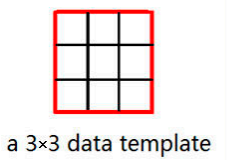

(a)

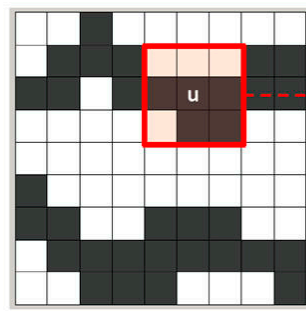

(b)

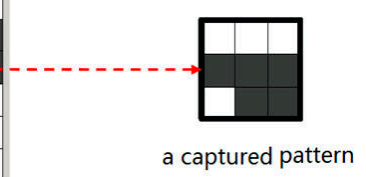

(c)

Figure 3. Illustration of a pattern captured by a $3 \times 3$ data template. (a) A $3 \times 3$ data template; (b) capture a pattern in a training image; (c) a captured pattern.

\subsubsection{Building a Pattern Dataset}

Normally a pattern dataset should be constructed before dimensionality reduction. For MPS simulation, all patterns should first be properly extracted from a training image. Each pattern means a point in high-dimensional space. A training image is scanned with $\tau_{D}$, and $\mathbf{t i}(\mathbf{u})$ is defined as a pattern centered at $\mathbf{u}$, and captured by $\tau_{D}$ in a training image. Let $t i\left(\mathbf{u}+\mathbf{h}_{\alpha}\right)$ be the state at $\mathbf{u}+\mathbf{h}_{\alpha}$, so ti( $\left.\mathbf{u}\right)$ can be defined as follows:

$$
\mathbf{t i}(\mathbf{u})=\left(t i\left(\mathbf{u}+\mathbf{h}_{1}\right), t i\left(\mathbf{u}+\mathbf{h}_{2}\right), \ldots, t i\left(\mathbf{u}+\mathbf{h}_{D}\right)\right) .
$$

Extract all patterns from a training image and let them be location-independent, so that one can get the $k$-th pattern:

$$
\operatorname{pat}_{k}=\left(\text { pat }_{k}\left(\mathbf{h}_{1}\right), \text { pat }_{k}\left(\mathbf{h}_{2}\right), \ldots, \text { pat }_{k}\left(\mathbf{h}_{D}\right)\right), k=1,2, \ldots, N_{\text {pat }},
$$

where $N_{\text {pat }}$ is the total number of captured patterns, and each pat $k_{k}\left(\mathbf{h}_{\alpha}\right)$ corresponds to $t i\left(\mathbf{u}+\mathbf{h}_{\alpha}\right)(\alpha=1$, $2, \ldots, D)$. Therefore, the pattern dataset patDb can be described:

$$
\text { patDb }=\left(\text { pat }_{1}, \text { pat }_{2}, \ldots, \text { pat }_{N p a t}\right),
$$

where each pat ${ }_{k}\left(k=1,2, \ldots, N_{\text {pat }}\right)$ means a point in high-dimensional space. Figure 4 a shows the process of $\tau_{D}$ (displayed with a $3 \times 3$ pixel grid) scanning over a gray-and-white training image. $\tau_{D}$ moves only one-pixel (or one-node) distance for each capture, from left to right in each row. Figure $4 \mathrm{~b}$ is the captured pattern dataset [24].

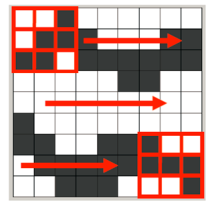

(a)

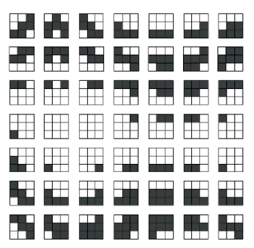

(b)

Figure 4. Illustration of building a pattern dataset of a training image. (a) Scan a training image; (b) a pattern dataset. 


\subsection{Step 2: Dimensionality Reduction of Patterns by ISOMAP}

Each pat $_{k}$ has $D$ elements, so it can be considered as a point in a $D$-dimensional space. Our plan of dimensionality reduction is to map each pat p $_{k}$ to a $d$-dimensional space $(d<<D)$, while the intrinsic relations and characteristics in pat $_{k}$ will be well maintained. Therefore, the whole process of dimensionality reduction can be depicted as follows: the pattern dataset patDb that includes $N_{\text {pat }}$ patterns pat $_{k}\left(k=1,2, \ldots, N_{\text {pat }}\right.$, pat $\left._{k} \in \mathbb{R}^{D}\right)$ will be mapped using ISOMAP from a $D$-dimensional space to a $d$-dimensional one, and then a new dataset $\mathbf{Y}\left(=\left(y_{1}, y_{2}, \ldots, y_{N_{p a t}}\right), y_{k} \in \mathbb{R}^{d}\right)$, is acquired. Each $\boldsymbol{y}_{k}$ has a much lower dimensionality, but the number of patterns remains unchanged after dimensionality reduction. The process of dimensionality reduction using ISOMAP is:

\subsubsection{Step 2.1. Construct Neighborhood Graph G of Patterns}

Compute all the Euclidean distances between all pairs of data points to be used as an approximation to geodesic distances. Define the Euclidean distance between pat $_{i}$ and pat $_{j}$ as $d_{E}\left(\right.$ pat $\left._{i}, \mathbf{p a t}_{j}\right)$ :

$$
d_{E}\left(\text { pat }_{i}, \mathbf{p a t}_{j}\right)=\sqrt{\sum_{\alpha=1}^{D}\left(\text { pat }_{i}\left(\mathbf{h}_{\alpha}\right)-\text { pat }_{j}\left(\mathbf{h}_{\alpha}\right)\right)^{2}},\left(i, j=1,2, \ldots, N_{\text {pat }}\right) .
$$

If pat $_{j}$ is one of the $k$ nearest neighboring patterns of pat $_{i}$, draw a line between pat $_{j}$ and pat $_{i}$ in G. By repeating this step, one can get the neighborhood graph of $\mathbf{p a t}_{i}$ by drawing lines between the remaining $k-1$ nearest neighboring patterns, and pat ${ }_{i}$. For each pat $_{i}\left(i=1,2, \ldots, N_{\text {pat }}\right)$, one can finally acquire the neighborhood graph $\mathrm{G}$.

\subsubsection{Step 2.2. Find Out the Shortest Path $d_{M}\left(\mathbf{p a t}_{i}, \mathbf{p a t}_{j}\right)$ between Two Patterns}

If $\mathbf{p a t}_{i}$ and $\mathbf{p a t}{ }_{j}$ are directly linked by only one line within a neighborhood, initialize $d_{M}\left(\mathbf{p a t}_{i}, \mathbf{p a t}_{j}\right)$ $=d_{E}\left(\mathbf{p a t}_{i}, \mathbf{p a t}_{j}\right) ;$ otherwise $d_{M}\left(\mathbf{p a t}_{i}, \mathbf{p a t}_{j}\right)=\infty$. Note that above $d_{M}\left(\mathbf{p a t}_{i}, \mathbf{p a t}_{j}\right)$ is only an initialization of distance, and may be replaced by a smaller value in the subsequent computation using the following Equation (6). For each $p=1,2, \ldots, N_{\text {pat }}$, define the shortest path as

$$
d_{M}\left(\text { pat }_{i}, \text { pat }_{j}\right)=\min \left\{d_{M}\left(\text { pat }_{i}, \text { pat }_{j}\right), d_{M}\left(\text { pat }_{i}, \text { pat }_{p}\right)+d_{M}\left(\text { pat }_{p}, \text { pat }_{j}\right)\right\} .
$$

The matrix of shortest path distances can be defined as $D_{M}=\left(d_{M}\left(\mathbf{p a t}_{i}, \mathbf{p a t}_{j}\right)\right)^{2}, i, j=1,2, \ldots$, $N_{\text {pat }}$. The whole procedure of computing the shortest path by Equation (6) can be considered as the well-known Floyd's algorithm that can acquire the shortest path in a weighted graph [30]. Because Equation (6) is a recursive procedure, " $d_{M}\left(\mathbf{p a t}_{i}, \mathbf{p a t}_{p}\right)+d_{M}\left(\mathbf{p a t}_{p}\right.$, pat $\left._{j}\right)$ " in Equation (6) can lead to $d_{M}\left(\mathbf{p a t}_{i}, \mathbf{p a t}_{p}\right)$ finding the shortest path between $\mathbf{p a t}_{i}$ and $\mathbf{p a t}_{p}$, and similarly, $d_{M}\left(\mathbf{p a t}_{p}, \mathbf{p a t}_{j}\right)$ finding the shortest path between pat $_{p}$ and pat ${ }_{j}$, respectively. Such recursive procedure continues, and finally the shortest path $d_{M}\left(\right.$ pat $_{i}$, pat $\left._{j}\right)$, can be obtained. Even if initially $d_{M}\left(\right.$ pat $\left._{i}, \mathbf{p a t}_{p}\right)=\infty$, or $d_{M}\left(\right.$ pat $\left._{p}, \mathbf{p a t}_{j}\right)=$ $\infty$, after limited times of recursive procedures, $d_{M}\left(\mathbf{p a t}_{i}, \mathbf{p a t}_{p}\right)$ and $d_{M}\left(\mathbf{p a t}_{p}, \mathbf{p a t}_{j}\right)$ can be obtained by Equation (6).

\subsubsection{Step 2.3. Compute the $d$-Dimensional Mapping}

Compute the following symmetrical matrix

$$
B=-\frac{1}{2}\left[I-\frac{1}{N_{p a t}} l \cdot l^{T}\right] D_{M}\left[I-\frac{1}{N_{p a t}} l \cdot l^{T}\right],
$$

where $I$ is an $N_{\text {pat }}$-dimensional identity matrix, and $l$ is an all-one $N_{\text {pat }}$-dimensional column vector. Compute $B^{\prime}$ s largest $d$ eigenvalues $\lambda_{1} \geq \lambda_{2} \geq \ldots \geq \lambda_{d}$, whose corresponding eigenvectors are $\alpha_{1}, \alpha_{2}$, $\ldots, \boldsymbol{\alpha}_{d}$. Define $\boldsymbol{\alpha}_{p}^{m}\left(m=1,2, \ldots, N_{p a t} ; p=1,2, \ldots, d\right)$ as the $m$-th component of $\boldsymbol{\alpha}_{p}$. Then, the target $d$-dimensional matrix is 


$$
\mathbf{Y}=\left(y_{1}, y_{2}, \ldots, y_{N_{p a t}}\right)^{T}=\left(\sqrt{\lambda_{1}} \alpha_{1}, \sqrt{\lambda_{2}} \alpha_{2}, \ldots, \sqrt{\lambda_{d}} \alpha_{d}\right),
$$

where $\boldsymbol{y}_{m}$ is the vector of the $m$-th component of each $\sqrt{\lambda_{p}} \boldsymbol{\alpha}_{p}$, i.e., $\boldsymbol{y}_{m}=\left(\sqrt{\lambda_{1}} \boldsymbol{\alpha}_{1}^{m}, \sqrt{\lambda_{2}} \boldsymbol{\alpha}_{2}^{m}, \ldots, \sqrt{\lambda_{d}} \boldsymbol{\alpha}_{d}^{m}\right)$. After mapping from patDb $=\left\{\right.$ pat $\left._{k}\right\}\left(\right.$ pat $\left._{k} \in \mathbb{R}^{D}, k=1,2, \ldots, N_{\text {pat }}\right)$ to $\mathbf{Y}=\left\{\boldsymbol{y}_{k}\right\}\left(\boldsymbol{y}_{k} \in \mathbb{R}^{d}, k=1,2, \ldots\right.$, $N_{\text {pat }}$ ), the dimensionality of patterns has been reduced from $D$ to $d$.

The dimension of the low-dimensional space, i.e., $d$, is a key parameter for ISOMAP: if the dimension is too small, important data features are overlapped onto the same dimension; if the dimension is too large, the projections become unstable. $d$ is derived by the maximum likelihood method in this paper (for more details, see Ref. [31]).

\subsection{Step 3: Classification of Patterns}

After the dimensionality reduction is finished, the mapping of patterns in low-dimensional space $\mathbb{R}^{d}$ should be classified. Any clustering method can be applied to the classification of patterns, such as partitioning methods, hierarchical methods, and density-based methods. The notion of a cluster, as found by different algorithms, varies significantly in its properties. There is no objectively "correct" clustering algorithm, but each one has its own application fields. The most appropriate clustering algorithm for a particular problem often needs to be chosen experimentally, unless there is a mathematical reason to prefer one cluster model over another [32]. The classical k-means is chosen as the clustering method in this paper. Given an input number of clusters, the k-means algorithm finds the optimal centroid of each cluster, then assigns each pattern to a specific cluster according to an input distance [33].

After being clustered by k-means, the whole $d$-dimensional space is divided into some small cells. Suppose $L$ and $l$ are, respectively, the total number and sequence number of non-empty cells. For each cell, a prototype $\operatorname{prot}^{(l)}\left(\mathbf{h}_{\alpha}\right)$ that has the same size with the data template is defined as the average of the pattern located at $\mathbf{h}_{\alpha}$ in the $l$-th cell:

$$
\operatorname{prot}^{(l)}\left(\mathbf{h}_{\alpha}\right)=\frac{1}{c_{l}} \sum_{i=1}^{c_{l}} T\left(\mathbf{u}_{i}^{(l)}+\mathbf{h}_{\alpha}\right), \alpha=1, \ldots, D ; l=1, \ldots, L,
$$

where $c_{l}$ is the number of patterns in the $l$-th cell; $\mathbf{u}_{i}^{(l)}\left(i=1, \ldots, c_{l}\right)$ is the center of a data template in the $l$-th cell; $T$ means a training image, and $T\left(\mathbf{u}_{i}^{(l)}+\mathbf{h}_{\boldsymbol{\alpha}}\right)$ is the state of a training image at $\mathbf{u}_{i}^{(l)}+\mathbf{h}_{\boldsymbol{\alpha}}$. The average vector of the $l$-th cell's prototype is

$$
\operatorname{prot}_{l}=\left(\operatorname{prot}^{(l)}\left(\mathbf{h}_{1}\right), \operatorname{prot}^{(l)}\left(\mathbf{h}_{2}\right), \ldots, \operatorname{prot}^{(l)}\left(\mathbf{h}_{D}\right)\right), l=1, \ldots, L,
$$

which is considered as a representative for all patterns in the $l$-th cell.

For example, Figure 5 is an example of separating a $d$-dimensional pattern space into some cells represented by some dotted-line surrounded regions, in which there is an empty cell, and each black point means a pattern in this space. Figure 6a shows a training image containing sand channels in a shale background; Figure $6 \mathrm{~b}$ shows 15 patterns belonging to one cell; Figure $6 \mathrm{c}$ is the average of all these 15 patterns, namely the prototype of this cell. 


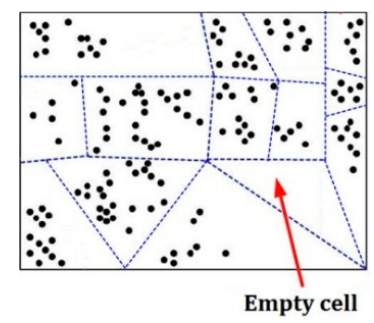

Figure 5. Illustration of separating pattern space into some cells.

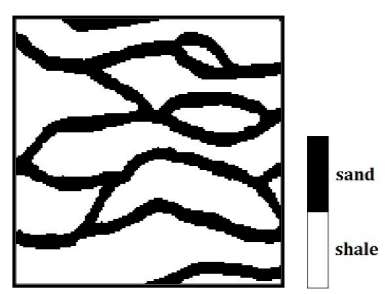

(a)

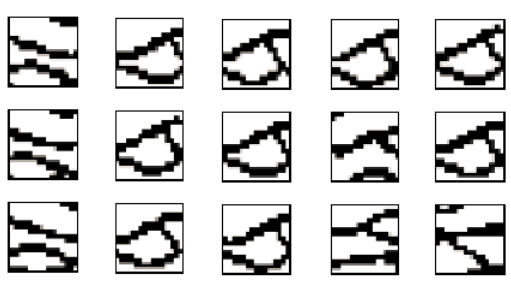

(b)

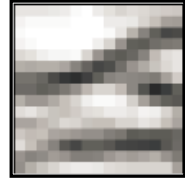

(c)

Figure 6. Illustration of a prototype. (a) A training image; (b) 15 patterns belonging to one cell; (c) a prototype of this cell.

\subsection{Step 4: Extraction of Patterns}

When extracting a pattern, this pattern is divided into the inner (frozen) part, and the outer part. Both parts are viewed as hard conditional data for the simulation of next node along the simulation path, but the difference is that only the nodes in the outer part will be resimulated. Normally the inner part is set to a square in the pattern; the remaining part contains the outer nodes. Figure 7 shows the two parts of a simulated pattern.

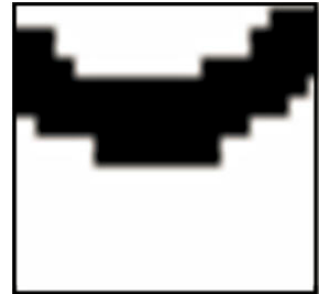

(a)

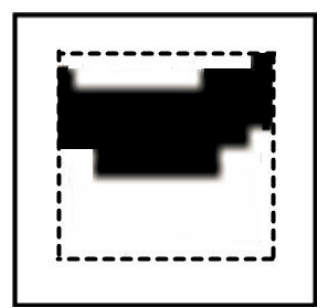

(b)

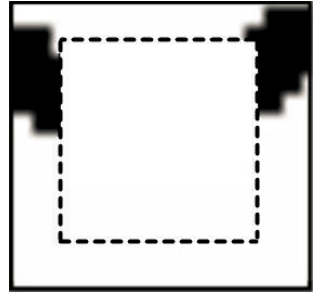

(c)

Figure 7. The inner part and outer part of a simulated pattern. (a) A simulated pattern; (b) the inner part of a simulated pattern (in the dotted line); (c) the outer part of a simulated pattern (outside the dotted line).

Considering $t$ as the data type, all nodes are divided into three categories: original known conditional data $(t=1)$, frozen data used as conditional data that will not be resimulated $(t=2)$, and simulated data that will be resimulated $(t=3)$. Each node is assigned with a different weight, $\omega(t)$, to measure its importance for computing distances according to its data type. At each node along the visiting path, the distance function $d i s\left(d\left(\mathbf{u}_{\alpha}\right)\right.$, $\left.\operatorname{prot}_{l}\right)$ representing distances between data event $d\left(\mathbf{u}_{\alpha}\right)$ (i.e., $d\left(\mathbf{u}+\mathbf{h}_{\alpha}\right)$ ), and $\mathbf{p r o t}_{l}$, is calculated by comparing each known nodal value in the data event with each prototype $\operatorname{prot}^{(l)}\left(\mathbf{h}_{\boldsymbol{\alpha}}\right)$ :

$$
\operatorname{dis}\left(d\left(\mathbf{u}_{\alpha}\right), \operatorname{prot}_{l}\right)=\sum_{\alpha=1}^{D} \omega(t)\left|d\left(\mathbf{u}+\mathbf{h}_{\alpha}\right)-\operatorname{prot}^{(l)}\left(\mathbf{h}_{\alpha}\right)\right|, t=1,2,3 ; l=1, \ldots, L,
$$


where $\omega(t)$ is the weight of nodes with $\omega(3) \leq \omega(2) \leq \omega(1)$. Clearly the original known conditional data have the highest weight. Note that the dimensionality reduction in Section 2.2 is only used for the classification of patterns in Section 2.3, and when extracting patterns, the distance in Equation (11) is calculated in the original space, but not the dimensionally reduced space.

In the super-resolution reconstruction of remote sensing images, coarse fraction information existing in low-resolution images is considered as soft data to constrain the reconstructions; hence the soft data always exist in the simulation procedure from the beginning to the end. The simulated nodes in the reconstructed region are both the results of super-resolution reconstruction, and the hard data for the unknown nodes in the subsequent simulation. It is seen that at the very beginning, since there are no any hard data in the region to be reconstructed, the reconstruction only depends on the coarse fraction information used as soft data. However, when the soft data and hard data are both available, consider the hard data event centered at $\mathbf{u}$ as $h d\left(\mathbf{u}_{\alpha}\right)$, and its "hard distance" with each prot $_{l}$ is

$$
\operatorname{dis}_{h d}^{l}=\operatorname{dis}\left(h d\left(\mathbf{u}_{\alpha}\right), \operatorname{prot}_{l}\right), l=1, \ldots, L,
$$

where $d i s_{h d}^{l}$ is the $l$-th hard distance, and $d i s(\cdot)$ means the distance calculation using Equation (11). Then the hard distance vector between $h d\left(\mathbf{u}_{\alpha}\right)$ and each prot $_{l}$ is

$$
\operatorname{Dis}_{h d}=\left(d i s_{h d}^{1}, d i s_{h d}^{2}, \ldots, d i s_{h d}^{L}\right) .
$$

As for soft data, similarly define the "soft distance" between the soft data event $s d\left(\mathbf{u}_{\alpha}\right)$ and each $\operatorname{prot}_{l}$ as:

$$
\operatorname{dis}_{s d}^{l}=\operatorname{dis}\left(s d\left(\mathbf{u}_{\alpha}\right), \operatorname{prot}_{l}\right), l=1, \ldots, L,
$$

where $d i s_{s d}^{l}$ is the $l$-th soft distance. Then the soft distance vector between $s d\left(\mathbf{u}_{\alpha}\right)$ and each prot $_{l}$ is

$$
\operatorname{Dis}_{s d}=\left(d i s_{s d}^{1}, d i s_{s d}^{2}, \ldots, d i s_{s d}^{L}\right)
$$

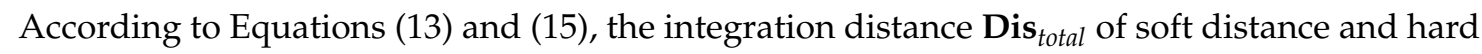
distance is

$$
\operatorname{Dis}_{t o t a l}=\operatorname{per}_{h d} \times \operatorname{Dis}_{h d}+\mu \times \operatorname{Dis}_{s d},
$$

where the distance vectors of $\mathbf{D i s}_{h d}$ and $\mathbf{D i s} s_{s d}$ should be normalized into [0,1] beforehand, per ${ }_{h d}$ is the percentage of known nodes in the current data event, $\mu(0 \leq \mu \leq 1)$ is related to the ratio of the area already reconstructed (called $A r e a_{r}$ ) to the total reconstruction area (called $A r e a_{T}$ ) and is defined as

$$
\mu=1-\frac{\text { Area }_{r}}{\text { Area }_{T}}
$$

Consider that

$$
\operatorname{dis}_{\text {total }}^{l}=\operatorname{per}_{h d} \times d i s_{h d}^{l}+\mu \times d i s_{s d}^{l}, l=1, \ldots, L
$$

then Dis total $_{\text {also is defined as }}$

$$
\begin{gathered}
\operatorname{Dis}_{\text {total }}=\left(d i s_{\text {total }}^{1}, \operatorname{dis}_{\text {total }}^{2}, \ldots, d i s_{\text {total }}^{L}\right) \\
=\left(\operatorname{per}_{h d} \times d i s_{h d}^{1}+\mu \times d i s_{s d}^{1} \operatorname{per}_{h d} \times d i s_{h d}^{2}+\mu \times d i s_{s d}^{2}, \ldots, \operatorname{per}_{h d} \times d i s_{h d}^{L}+\mu \times d i s_{s d}^{L}\right) .
\end{gathered}
$$

One can achieve the minimum among $d i s_{\text {total }}^{1}, d i s_{\text {total }}^{2} \ldots, d i s_{\text {total }}^{L}$. Let $R$ be the sequence number

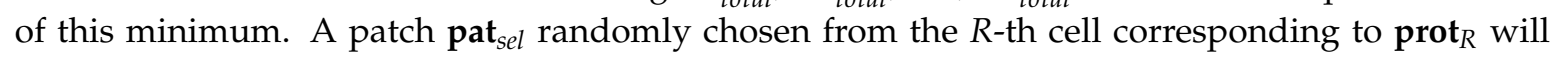
be pasted to the region to be simulated, except the frozen locations, and locations corresponding to original known hard data. Then, freeze some inner nodes that will not be resimulated, and move to the next node in the simulation path, until all the nodes are simulated. 
Note that coarse fraction information from low-resolution images is used as soft data, and this situation exists in the whole reconstruction, but hard data are not always existent. First, the super-resolution reconstruction proceeds with only soft data but hard data are not existent, so the distance computation in Equation (16) is only the calculation conditioned to the coarse fraction information to obtain $\mathbf{D i s}_{s d}$. When the reconstruction continues, more simulated nodes (pixels) are available to be hard data. Then Equation (16) becomes a real combination of calculating Dis $h d$ and Dis $_{s d}$, meaning using soft data and hard data simultaneously.

\section{The General Procedure of the Proposed Method}

After detailed explanations for the above four steps, the procedure of our method for super-resolution reconstruction of remote sensing images is summarized as follows:

Step 1. Construct a pattern dataset patDb by using a data template to scan a training image (or some training images).

Step 2. Perform the dimensionality reduction using ISOMAP.

Step 3. Perform classification of patterns using a clustering method (e.g., k-means) in low-dimensional space.

Step 4. Define a random path visiting all nodes in the simulation grid for super-resolution reconstruction. At each node along the path, determine a prototype $\operatorname{prot}_{R}$ closest to current data event by comparing the distance between current data event and each prototype using Equation (16), in which the distance calculation combines the distances of soft data (coarse fraction data from low-resolution images) and hard data (nodes already simulated). Afterwards, randomly select a pattern from the R-th cell, and copy this pattern to the region to be simulated; then, move to next node in this path until all the nodes are simulated.

The flow chart of our method is shown in Figure 8. 


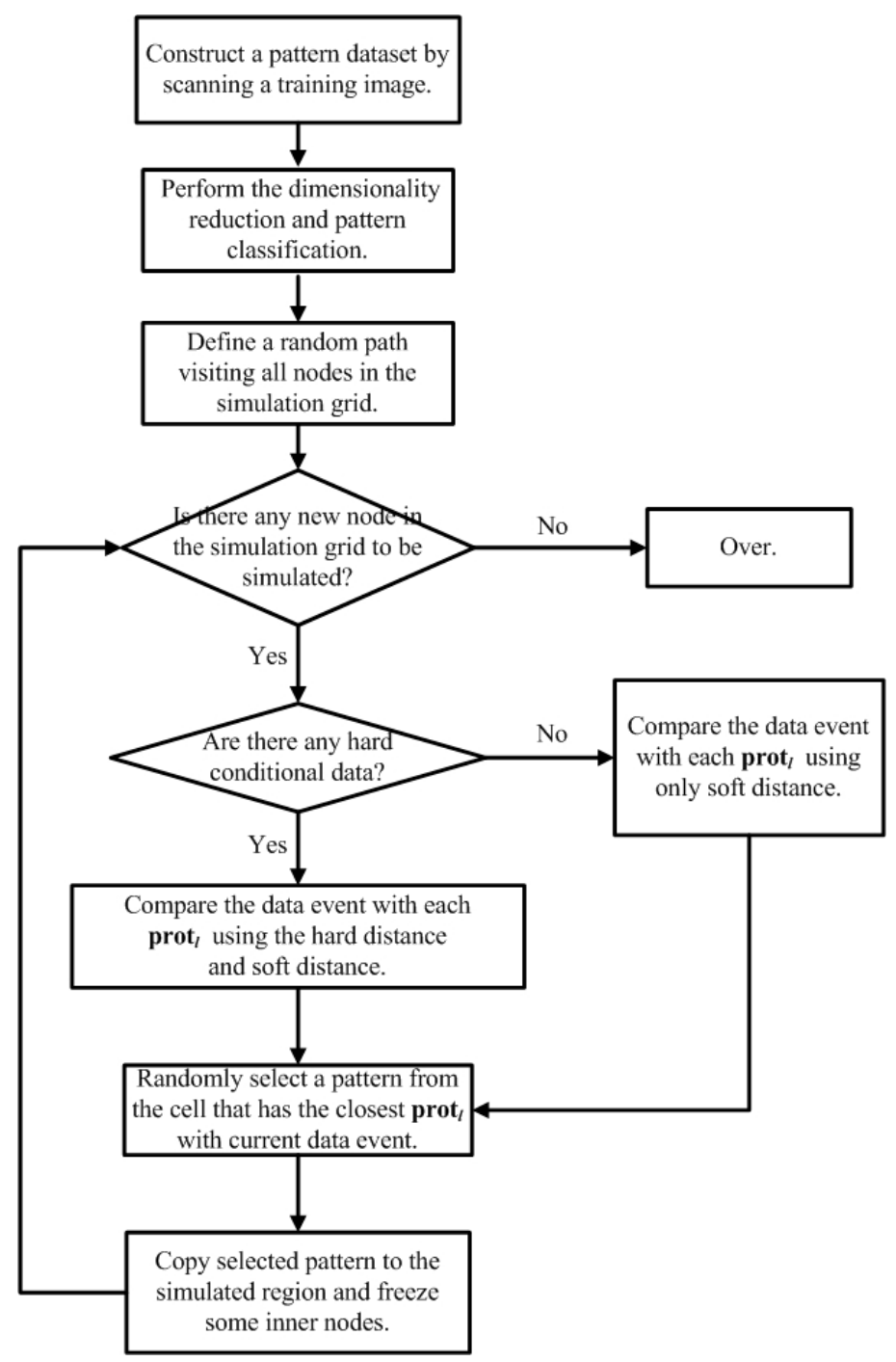

Figure 8. Flow chart of the proposed method.

\section{Experimental Results and Analyses}

\subsection{Evaluation Method of Reconstruction Quality}

MPS reconstruction is quite different from the non-stochastic reconstruction methods when evaluating reconstruction quality. When reconstructing the high-resolution remote sensing images from low-resolution images, MPS generates multiple stochastic results that provide an assessment of uncertainty regarding the true remote sensing images, as opposed to the single image solution frequently adopted in practice. Hence, it is impossible to achieve exactly the same simulated results with the training image when using MPS. MPS captures the features of a training image by copying its statistically intrinsic structures. It does not require the stochastic simulated results to be completely identical with the training image.

A variogram can be used to measure the similarity between simulated results and the training image, which is also the correlation between any two points in space when their separation increases, so it is used for evaluating the super-resolution reconstruction of remote sensing images. A variogram relates a variable at an unsampled location from its near locations, which is

$$
\gamma(\mathbf{h})=\frac{1}{2} E[Z(\mathbf{u})-Z(\mathbf{u}+\mathbf{h})]^{2},
$$


where $Z(\mathbf{u})$ and $Z(\mathbf{u}+\mathbf{h})$, respectively, denote the estimators of the variable $Z$ at locations $\mathbf{u}$ and $\mathbf{u}+\mathbf{h}$; $\mathbf{h}$ is the lag distance; E means mathematical expectation [34].

\subsection{Case Study}

The experiments were run on a computer with an Intel core i3-4160T (a 3.1 GHz CPU) and 4 GB memory. The programming software was Matlab 2010. For parameters in Equation (11), $\omega(1)=0.5$, $\omega(2)=0.3$, and $\omega(3)=0.2$. The template size is $17 \times 17$ pixels, and its inner part is $9 \times 9$ pixels. The capability of the proposed method to reproduce the super-resolution information of remote sensing images is demonstrated, hereafter, via a few categorical and continuous reconstructions of synthetic and real cases. The main objective is to illustrate that the prior structural features existing in the training images can be successfully generated in the super-resolution remote sensing images by our method. Note that these case studies are not to make the most locally accurate super-resolution remote sensing images for the particular region, but to generate multiple super-resolution reconstructions of remote sensing images from the available coarse resolution information, which can be used for some uncertainty assessment of risk evaluation, or decision making, that will not be discussed in this paper.

\subsubsection{D Categorical Simulation}

Super-resolution reconstructions for categorical variables are tested in this section. It should be noted that the training images and reference images considered in this categorical case are simply chosen for illustrative purposes. In the real-world applications, the true remote sensing images may be more complex, and contain more kinds of variables or classes. Consider two synthetic binary reference images, shown in Figure 9a,b, each of size $150 \times 150$ pixels. These images are hereafter called Cases $\# 1$, and \#2, respectively. Case \#1 shows typical curvilinear shapes in a fluvial region. Case \#2 shows a region filled with round shapes (including bigger ellipses and smaller circles), and few speckles. Set the black pixels (i.e., pixels representing the curvilinear shapes in Case \#1, and round shapes and speckles in Case \#2) to attribute value 1, and the gray pixels (representing the background in above two cases) to attribute value 0 . The average of Figure 9a,b are 0.27 and 0.30 , respectively. The lower dimension $d$, is set to 3 for both Case \#1 and Case \#2, derived by the maximum likelihood method.

The reference images of Figure 9 were then upscaled into two coarse fraction images using two different coarse resolution ratios. For each coarse pixel, the corresponding fractions were computed as the linear average of the fine pixel values within that coarse pixel. The coarse fraction images for Cases \#1 and \#2, with different resolutions computed from each reference image, are shown in Figures 10 and 11, respectively. These coarse fraction images in Figure 10 were computed by linearly averaging the reference images in Figure 9 into blocks of $25 \times 25$ fine pixels each, creating images of $6 \times 6$ coarse pixels. Similarly, Figure $11 a, b$ were linear averages of the reference images in Figure 9, with blocks of $15 \times 15$ fine pixels each, creating images of $10 \times 10$ coarse pixels. For Figures 10 and 11 , each coarse pixel is retrogressively comprised of $25 \times 25=625$, and $15 \times 15=225$ fine pixels, and the whole image is progressively comprised of $6 \times 6=36$, and $10 \times 10=100$ coarse pixels. Obviously Figure 11 has higher resolution than Figure 10.

Two training images for Cases \#1 and \#2 with $250 \times 250$ pixels are shown in Figure 12 . The prior structural models contained in the training images can constrain the reconstruction. Note that the training images and reference images have different sizes and are not identical, but they share similar patterns. In the following super-resolution reconstruction, our method was tested with the aid of coarse fraction information, meaning Figures 10a and 11a were used as soft data, respectively, for Case \#1 to reproduce the super-resolution reconstructed images with coarse fractions of two different resolutions $(6 \times 6$ and $10 \times 10$ coarse pixels), and the prior structural features were extracted from Figure 12a, that was used as the training image; also Figures $10 \mathrm{~b}$ and $11 \mathrm{~b}$ were used for the super-resolution reconstruction of Case \#2, with two coarse resolutions $(6 \times 6$ and $10 \times 10$ coarse pixels $)$ respectively, and accordingly Figure $12 \mathrm{~b}$ was the training image. 
One hundred super-resolution realizations for Cases \#1 and \#2 were respectively generated, with different coarse fraction data, and some of them are shown in Figures 13a-d and 14a-d. It is seen that each reconstruction reproduces the structural features in Figure 9. However, the images generated with higher-resolution coarse fraction images (i.e., Figure 11a,b) clearly have better reconstruction quality. For example, in Figure 14a,b, the curvilinear shapes have fewer intervals, but in Figure 13a,b, the shapes are more disconnected. It also can be observed that in Figure $14 \mathrm{c}, \mathrm{d}$, each ellipsis and circle are more complete and isolated, but many ellipses and circles are overlapped and irregular in Figure 13c,d.

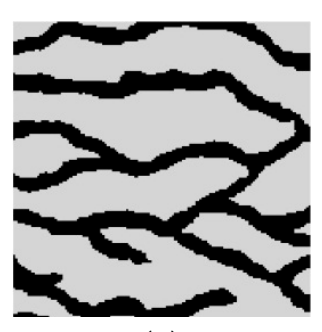

(a)

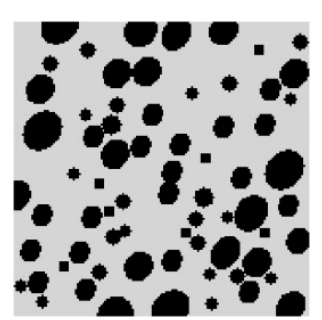

(b)

Figure 9. Reference images of size $150 \times 150$ pixels, labeled Cases \#1 and \#2. (a) Case \#1; (b) Case \#2.

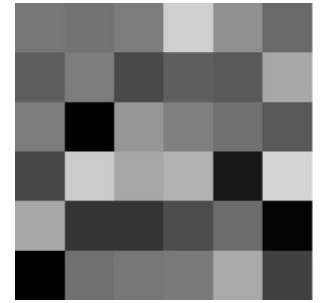

(a)

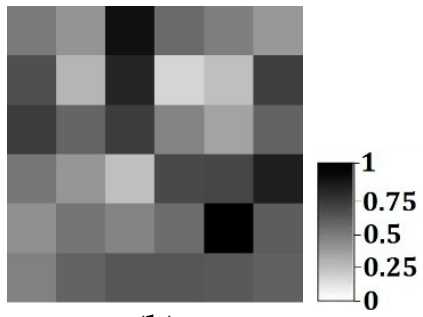

(b)

Figure 10. Coarse fractions computed from the reference images in Figure 9. Each coarse pixel contains $25 \times 25$ fine pixels, creating images of $6 \times 6$ coarse pixels. (a) Case \#1; (b) Case \#2.

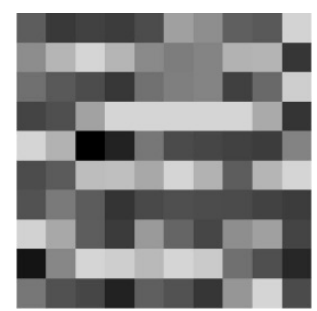

(a)

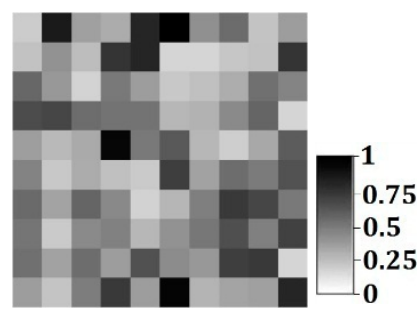

(b)

Figure 11. Coarse fractions computed from the reference images in Figure 9. Each coarse pixel contains $15 \times 15$ fine pixels, creating images of $10 \times 10$ coarse pixels. (a) Case \#1; (b) Case \#2. 


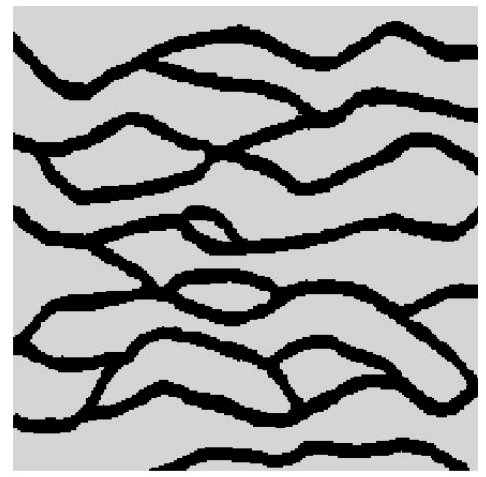

(a)

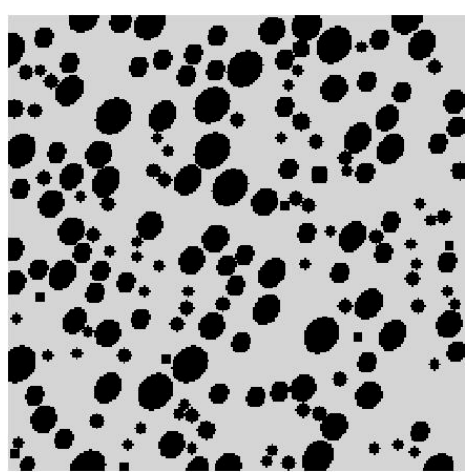

(b)

Figure 12. Training images of $250 \times 250$ pixels for Cases \#1 and \#2. (a) Case \#1; (b) Case \#2.

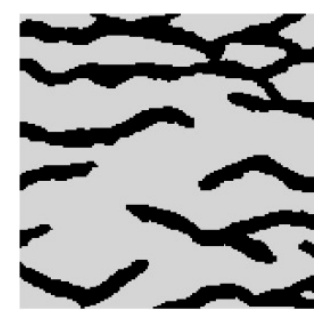

(a)

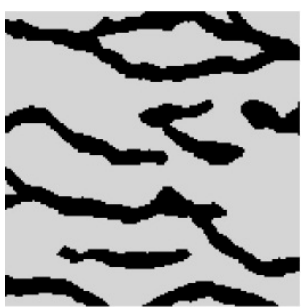

(b)

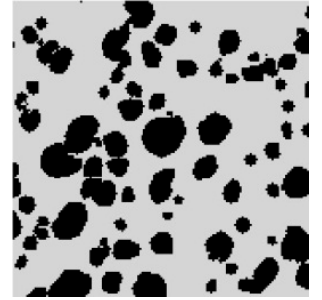

(c)

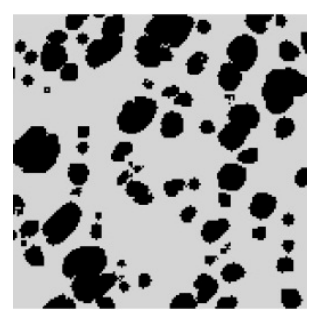

(d)

Figure 13. Super-resolution realizations for each of the reference cases using corresponding training images and coarse fraction data of Figure 10. (a) Case \#1, Realization \#1; (b) Case \#1, Realization \#2; (c) Case \#2, Realization \#1; (d) Case \#2, Realization \#2.

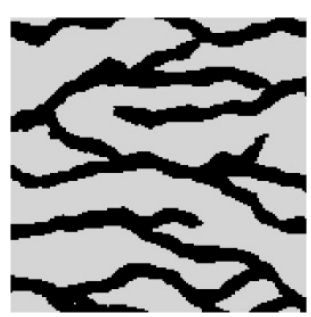

(a)

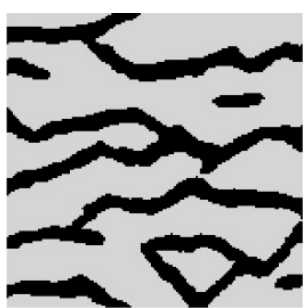

(b)

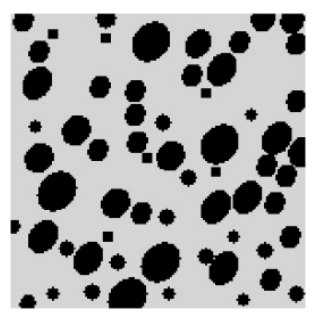

(c)

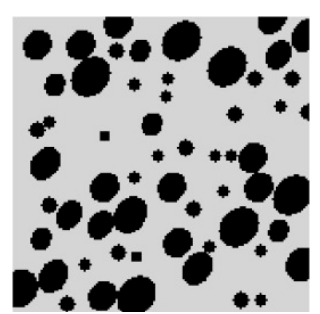

(d)

Figure 14. Super-resolution realizations for each of the reference cases using corresponding training images and coarse fraction data of Figure 11. (a) Case \#1, Realization \#1; (b) Case \#1, Realization \#2; (c) Case \#2, Realization \#1; (d) Case \#2, Realization \#2.

The coarse fraction reproduction is corroborated in Figures 15 and 16, respectively, where the averages of coarse fraction images computed from the super-resolution reconstructed images are plotted against the coarse fraction data of reference images (a $45^{\circ}$ line indicates good reproduction). The fluctuation of all the points near the $45^{\circ}$ line indicates the good reproduction of coarse fractions in reconstructed images, but currently it is hard to find out which situation, meaning use of Figures 13 and 14 fits the reference image better, by observing their distribution around the $45^{\circ}$ line. Another measure of spatial statistical reproduction by variogram curves is given in Figures 17 and 18, where the average variograms calculated from 100 super-resolution realizations for each case are compared with the variograms of corresponding training images, demonstrating that coarse fractions with higher resolution enhance the quality of super-resolution reconstruction. 


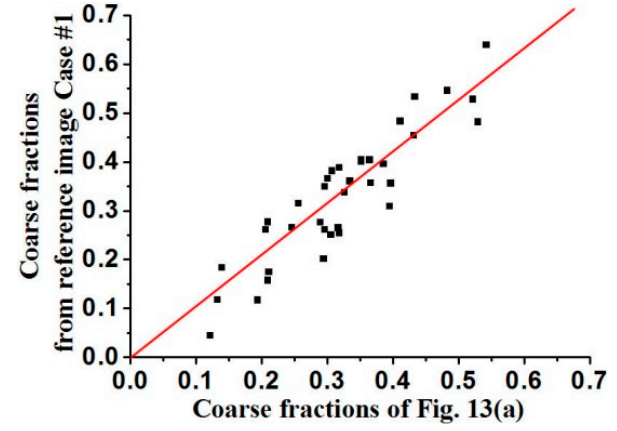

(a)

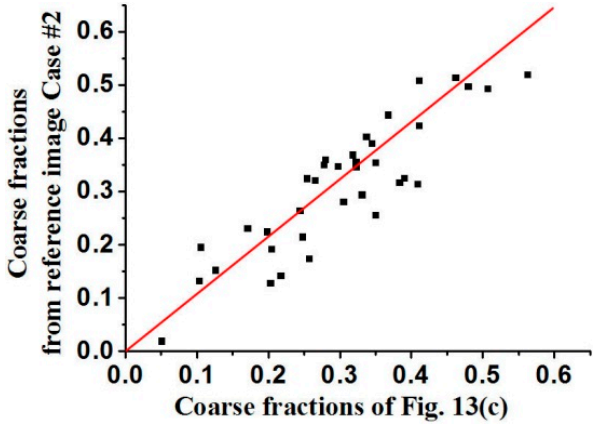

(b)

Figure 15. Coarse fraction reproduction of the super-resolution reconstruction images of Figure 13a,c. A $45^{\circ}$ line indicates a perfect reproduction. (a) Case \#1, Realization \#1; (b) Case \#2, Realization \#1.

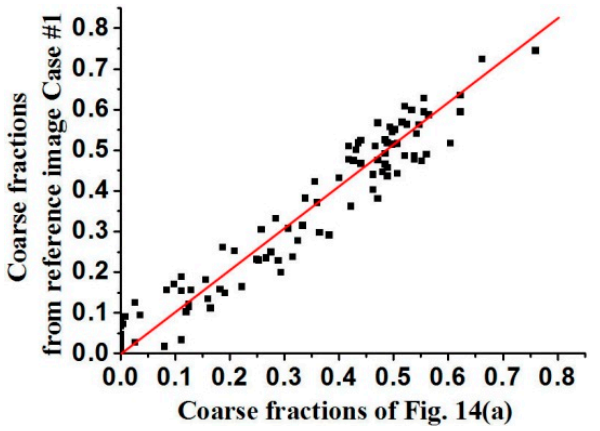

(a)

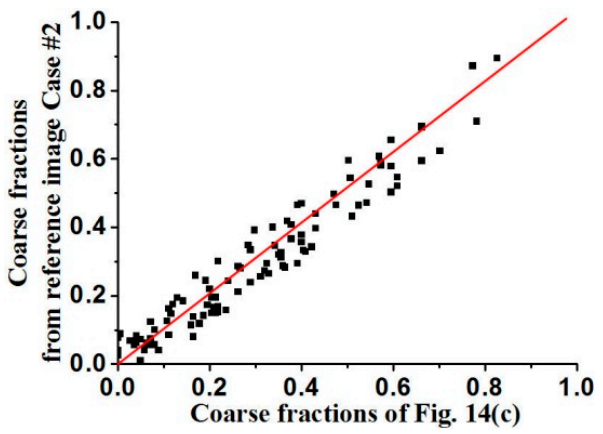

(b)

Figure 16. Coarse fraction reproduction of the super-resolution reconstruction images of Figure 14a,c. A $45^{\circ}$ line indicates a perfect reproduction. (a) Case \#1, Realization \#1; (b) Case \#2, Realization \#1.

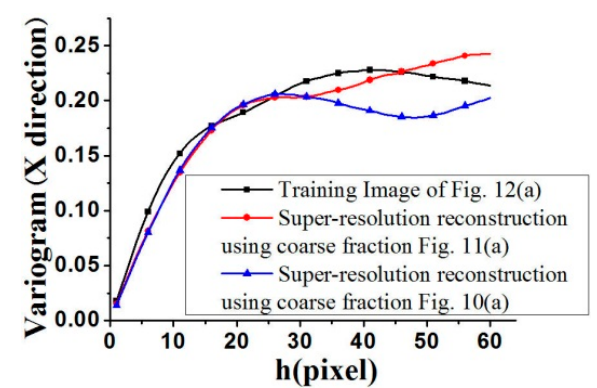

(a)

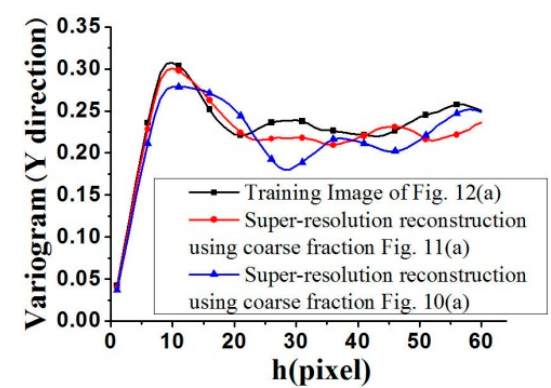

(b)

Figure 17. Variogram of the training image (Figure 12a) and average variograms of 100 reconstructions using coarse fractions (Figures 10a and 11a) in the $\mathrm{X}$ and $\mathrm{Y}$ directions. (a) $\mathrm{X}$ direction; (b) $\mathrm{Y}$ direction. 


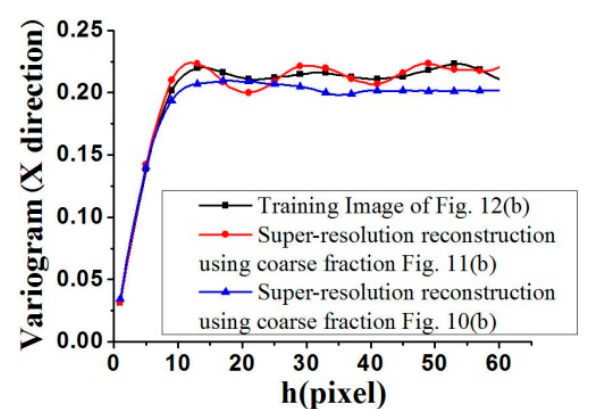

(a)

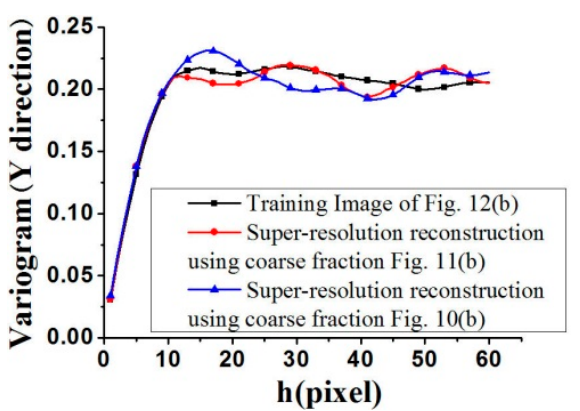

(b)

Figure 18. Variogram of the training image (Figure 12b) and average variograms of 100 reconstructions using coarse fractions (Figures $10 \mathrm{~b}$ and $11 \mathrm{~b}$ ) in the $\mathrm{X}$ and $\mathrm{Y}$ directions. (a) $\mathrm{X}$ direction; (b) $\mathrm{Y}$ direction.

\subsubsection{D Continuous Simulation}

In the last section, the tests are for categorical variables; in what follows, the tests are performed for continuous variables. Consider two remote sensing images as reference images acquired from Landsat TM data (band 2, satellite reflectance values, acquired in June 1996) of an area in the Changjiang River Delta, East China. The reference remote sensing images hereafter called Cases \#3 and \#4, are shown in Figure 19. Each image includes $512 \times 512$ fine resolution pixels, with each pixel of dimension $30 \mathrm{~m}$ $\times 30 \mathrm{~m}$. It is seen that Case \#3 contains some scattered forests (deep-colored region), a lake in the center of image, and some farmlands at the left-bottom corner; Case \#4 has many rural rectangular farmlands, and a river with two branches crossing the whole image. Both cases have some continuous roads. These typical landmarks in Cases \#3 and \#4 will be used to evaluate the reconstruction quality.

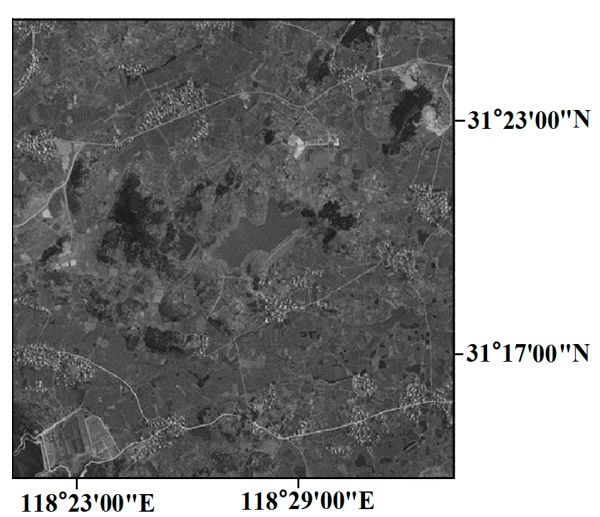

(a)

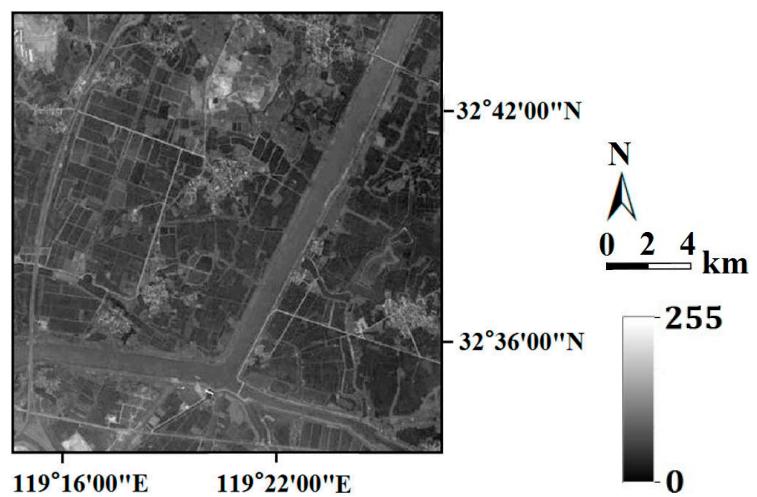

(b)

Figure 19. Reference images of $512 \times 512$ pixels, labeled Cases \#3 and \#4. (a) Case \#3; (b) Case \#4.

Similar to Cases \#1 and \#2, the above reference images were then upscaled into two coarse fraction images using two different resolutions by computing the linear average of the fine pixel values within a coarse pixel. The coarse fraction images for Cases \#3 and \#4, with different resolutions, are shown in Figures 20 and 21, respectively. The coarse fraction images in Figure 20 were computed by linearly averaging the reference images into blocks of $8 \times 8$ fine pixels each, creating images of $64 \times 64$ coarse pixels. Figure 21 is composed of linear averages of Cases \#3 and \#4, with blocks of $4 \times 4$ fine pixels each, creating images of $128 \times 128$ coarse pixels. Obviously, from Figure 20 to Figure 21, each coarse pixel is retrogressively comprised of $8 \times 8=64$, and $4 \times 4=16$ fine pixels, and the whole image is progressively comprised of $64 \times 64=4096$ and $128 \times 128=16,384$ coarse pixels.

Two training images of $800 \times 600$ pixels also acquired from Landsat TM data (band 2, satellite reflectance values, acquired in June 1996), are assigned to the two cases of Figure 19, respectively. 
Although the training images and reference images are not identical, they have some similar patterns, especially the previously mentioned landmarks, e.g., the lakes, forests, farmlands, rivers and roads can be observed in the training images. In the following reconstruction, Figures 20a and 21a with two different coarse resolutions ( $64 \times 64$ and $128 \times 128$ coarse pixels) were used as soft data, respectively, for Case \#3, to reproduce the super-resolution reconstructed images; their corresponding training image was Figure 22a. Figures 20b and 20b with two coarse resolutions $(64 \times 64$ and $128 \times 128$ coarse pixels) were used as soft information, respectively, for the super-resolution reconstruction of Case \#4, and accordingly, Figure $22 \mathrm{~b}$ was the training image.

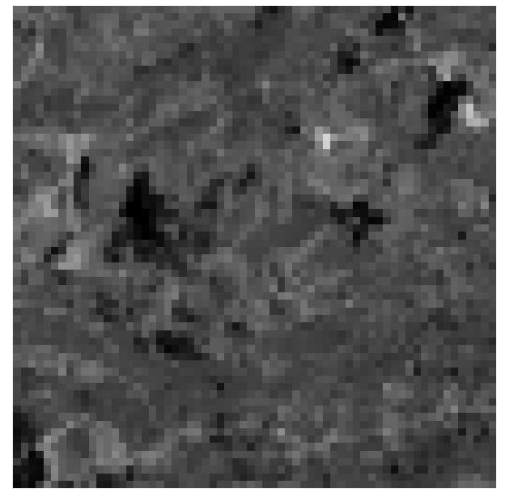

(a)

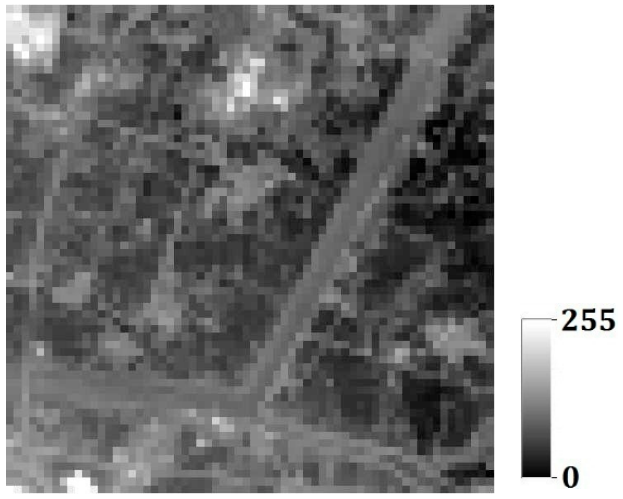

(b)

Figure 20. Coarse fractions computed from the reference images in Figure 19. Each coarse pixel contains $8 \times 8$ fine pixels, creating images of $64 \times 64$ coarse pixels. (a) Case \#3; (b) Case \#4.

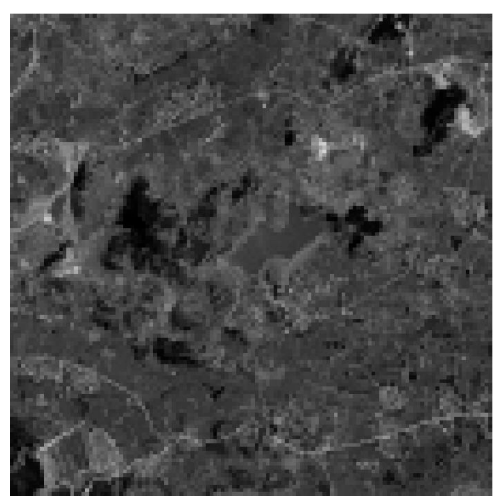

(a)

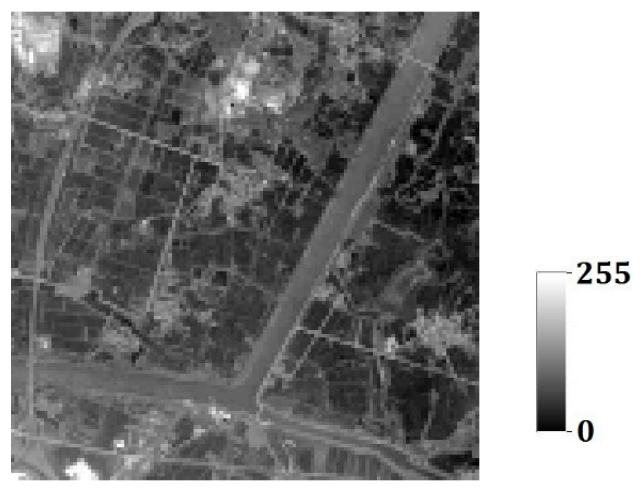

(b)

Figure 21. Coarse fractions computed from the reference images in Figure 19. Each coarse pixel contains $4 \times 4$ fine pixels, creating images of $128 \times 128$ coarse pixels. (a) Case \#3; (b) Case \#4.

The lower dimension $d$ is set to 4 for both Case \#3 and Case \#4, derived by the maximum likelihood method. One hundred super-resolution realizations for Cases \#3 and \#4 were respectively generated with different coarse fractions, and some of them are shown in Figures 23a-d and 24a-d. It is clearly seen that the images generated using higher-resolution coarse fraction images (i.e., Figure 21a,b) have better reconstruction quality. For example, in Figure 24a,b, the left-bottom farmlands can still be clearly observed, but almost disappear in Figure 23a,b; the rivers are more complete in Figure 24c,d than in Figure $23 \mathrm{c}$,d, especially the right-bottom part of rivers. However, in all reconstructed images, the roads are heavily disconnected, or not reproduced, because the prior information of roads in the training images is not quite adequate for reconstruction. After all, there are only a small number of roads in the training images. 

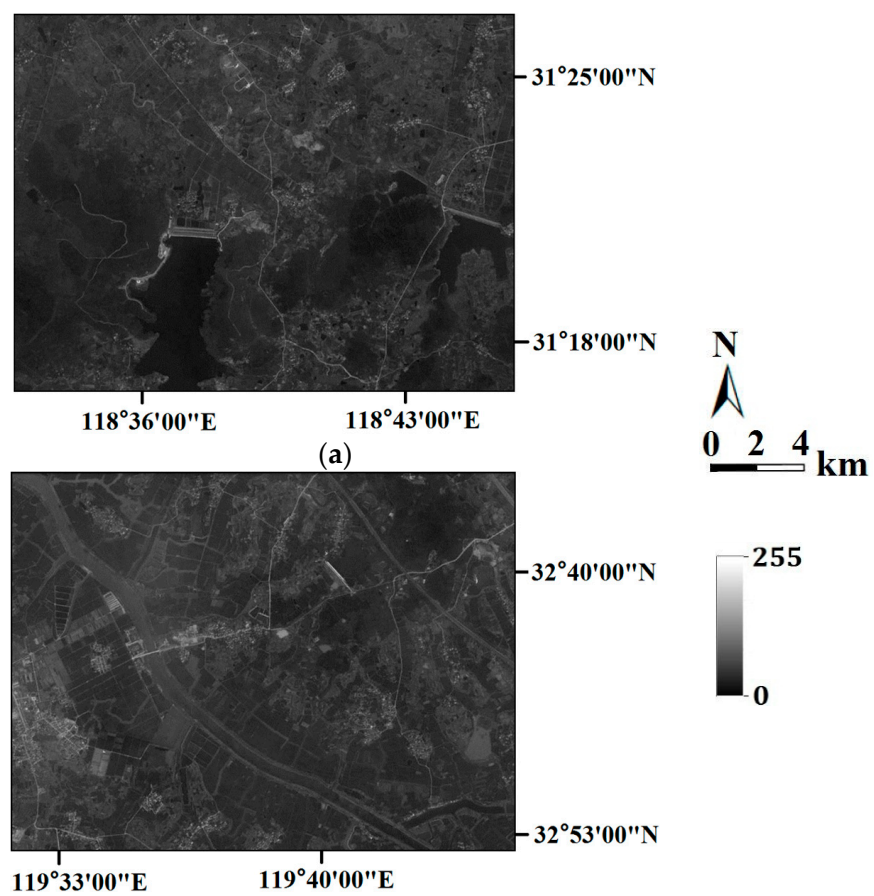

(b)

Figure 22. Training images of $800 \times 600$ pixels for Cases \#3 and \#4, respectively. (a) Case \#3; (b) Case \#4.

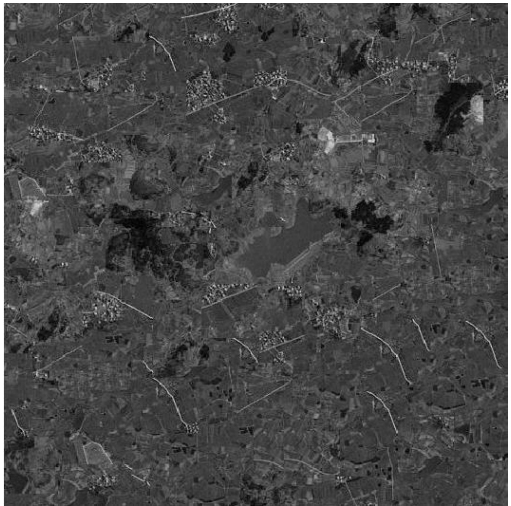

(a)

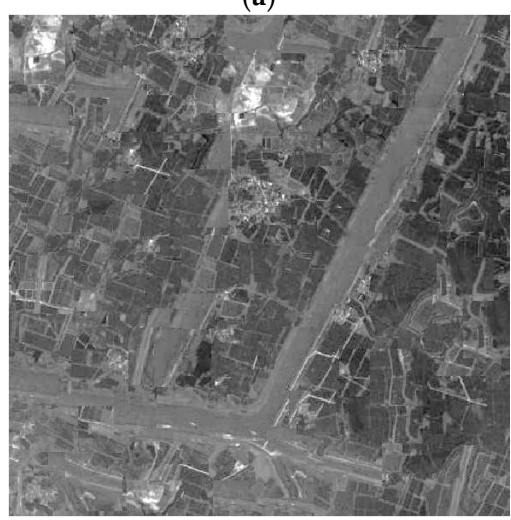

(c)

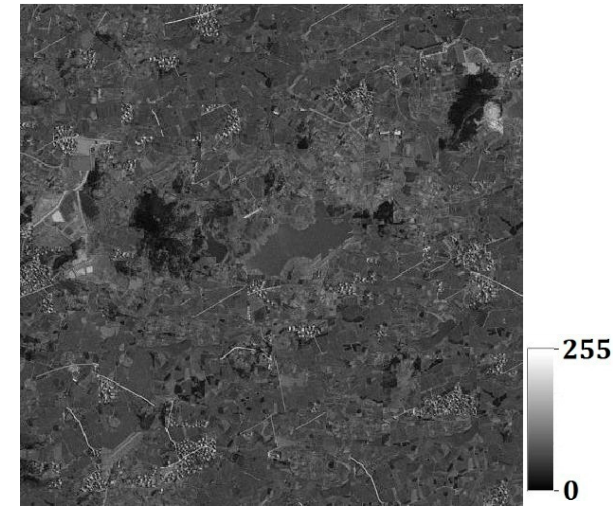

(b)

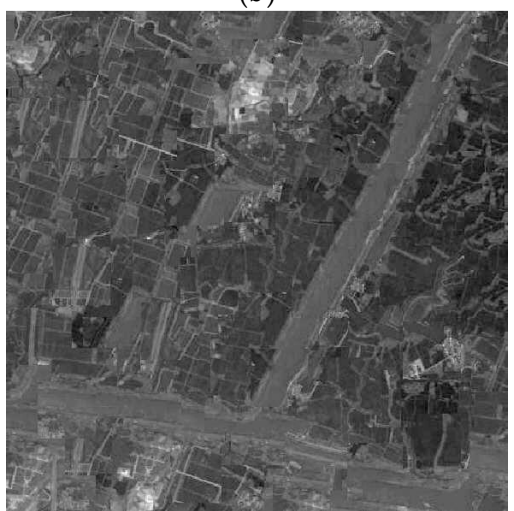

(d)

Figure 23. Super-resolution realizations for each of the reference cases using corresponding training images and coarse fractions of Figure 20. (a) Case \#3, Realization \#1; (b) Case \#3, Realization \#2; (c) Case \#4, Realization \#1; (d) Case \#4, Realization \#2. 


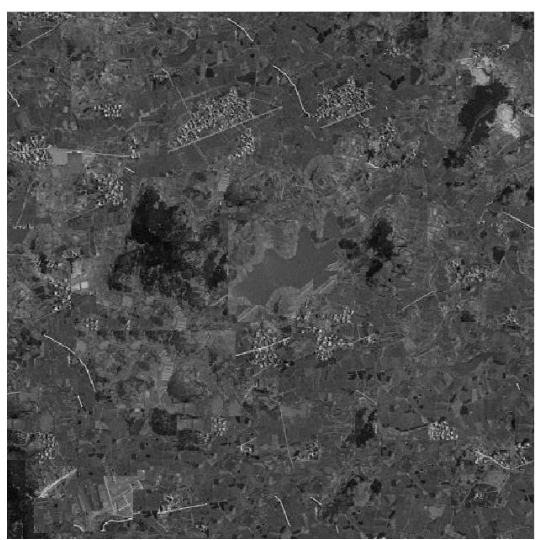

(a)

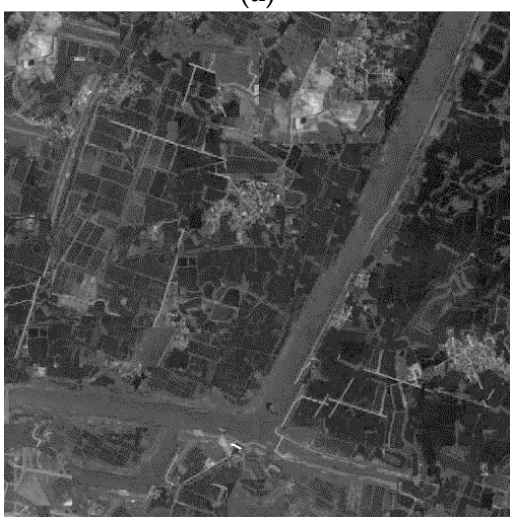

(c)

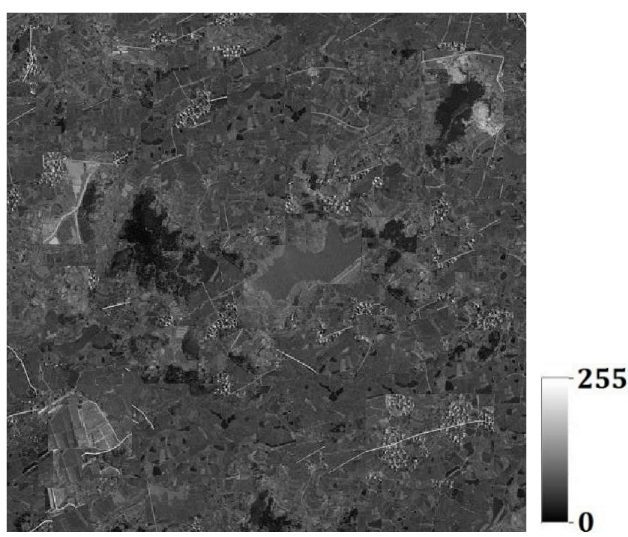

(b)

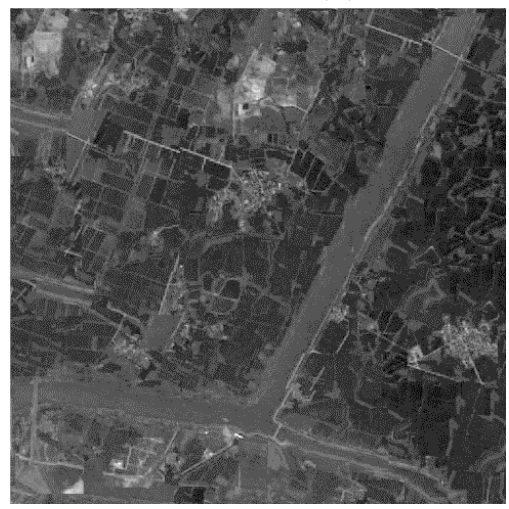

(d)

Figure 24. Super-resolution realizations for each of the reference cases using corresponding training images and coarse fractions of Figure 21. (a) Case \#3, Realization \#1; (b) Case \#3, Realization \#2; (c) Case \#4, Realization \#1; (d) Case \#4, Realization \#2.

In Figures 25 and 26, the averages of coarse fraction images computed from the super-resolution reconstructed images are plotted against the coarse fraction data of reference images, showing the better reproductivity of super-resolution reconstructed results using higher-resolution coarse fractions. In Figures 27 and 28, the average standardized variograms calculated from 100 super-resolution realizations for Cases \#3 and \#4 are compared with the standardized variograms of corresponding training images, also demonstrating that coarse fractions with higher resolution enhance the reconstruction quality.

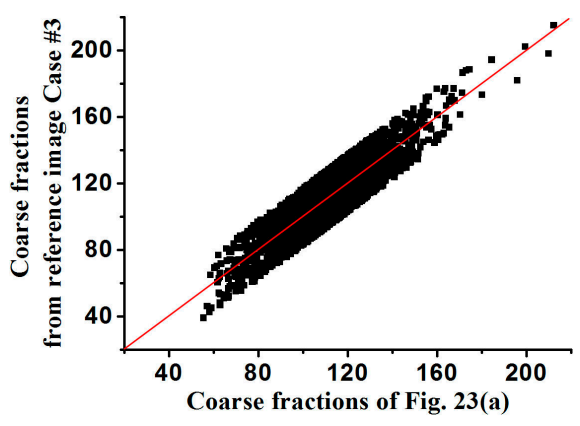

(a)

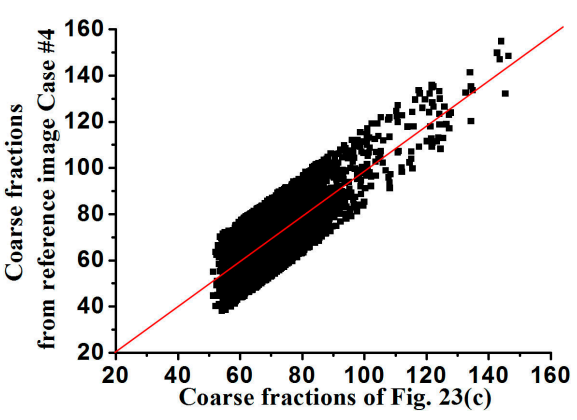

(b)

Figure 25. Coarse fraction reproduction of the super-resolution reconstruction images of Figure 23a,c. A $45^{\circ}$ line indicates a perfect reproduction. (a) Case \#3, Realization \#1; (b) Case \#4, Realization \#1. 


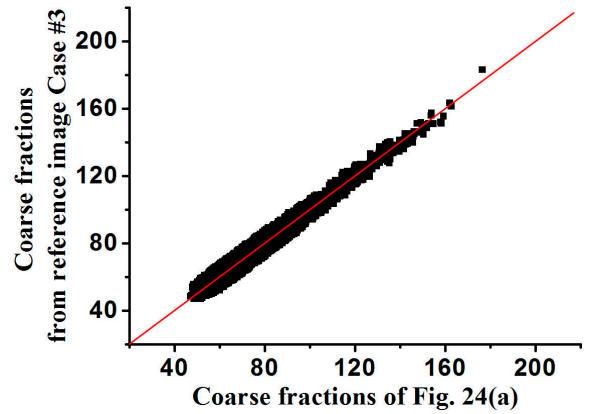

(a)

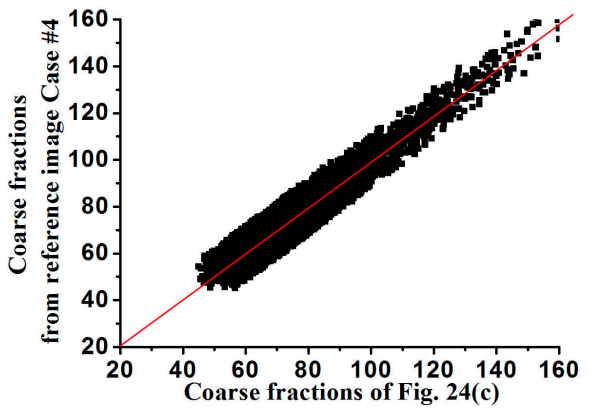

(b)

Figure 26. Coarse fraction reproduction of the super-resolution reconstruction images of Figure 24a,c. A $45^{\circ}$ line indicates a perfect reproduction. (a) Case \#3, Realization \#1; (b) Case \#4, Realization \#1.

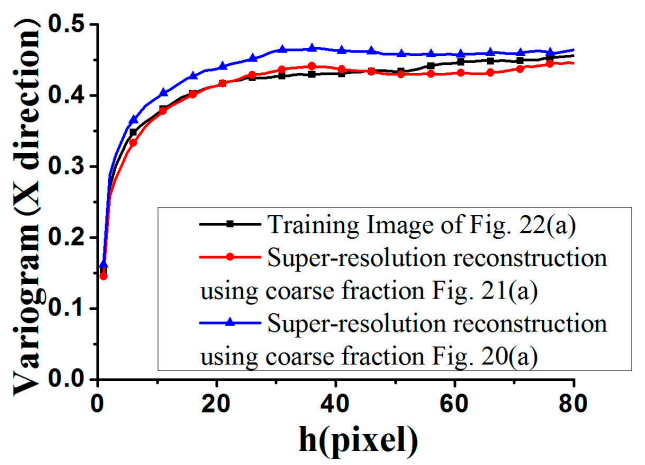

(a)

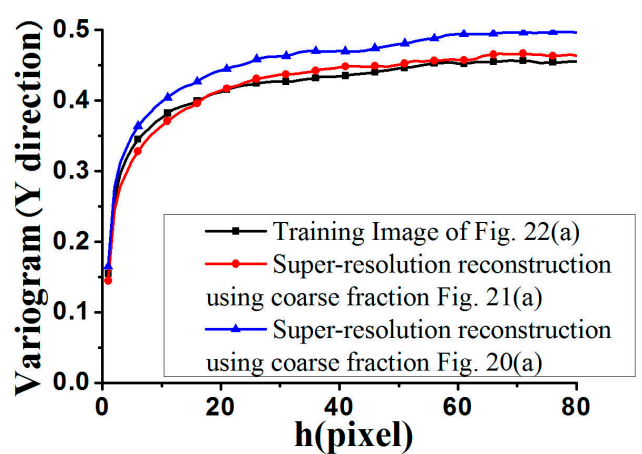

(b)

Figure 27. Standardized variogram of the training image (Figure 22a) and standardized average variograms of 100 reconstructions using coarse fractions (Figures 21a and 20a) in the X and Y directions. (a) X direction; (b) Y direction.

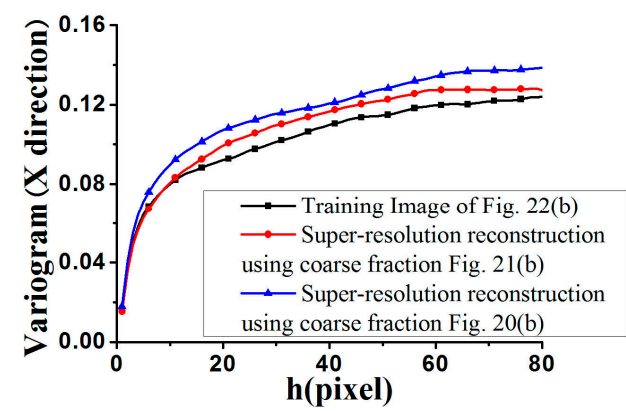

(a)

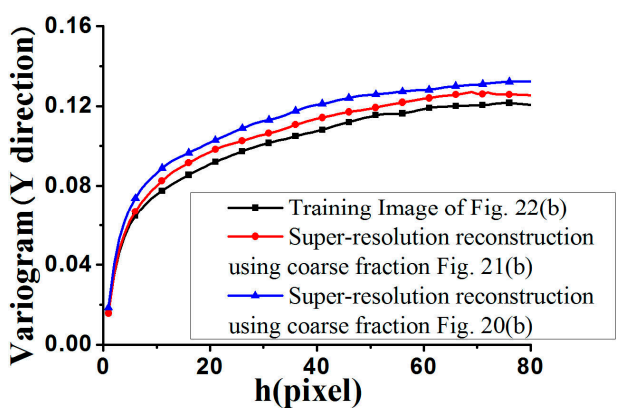

(b)

Figure 28. Standardized variogram of the training image (Figure 22b) and standardized average variograms of 100 reconstructions using coarse fractions (Figures $21 \mathrm{~b}$ and $20 \mathrm{~b}$ ) in the $\mathrm{X}$ and $\mathrm{Y}$ directions.

(a) X direction; (b) Y direction.

\subsection{Results of FILTERSIM and DISPAT}

As mentioned previously, FILTERSIM and DISPAT are two typical MPS methods heavily depended upon for linear dimensionality reduction $[23,24]$. To compare them with our method, some tests were performed using FILTERSIM and DISPAT to achieve super-resolution reconstruction, based on the training image of Figure 22b, and coarse fraction data of Figure 20b. The low dimensionality of FILTERSIM, and that of DISPAT, are both 6 . One hundred reconstructions by FILTERSIM and DISPAT 
were achieved, and some of them are shown in Figures 29 and 30. The standardized variograms are shown in Figure 31. The simulation time, and maximum memory, are displayed in Table 1. Clearly, the proposed method has shown its advantage over FILTERSIM and DISPAT in reconstruction quality and CPU time, but the memory costs are quite similar for the three methods.

Table 1. CPU time and maximum memory for 100 reconstructions using the proposed method, FILTERSIM and DISPAT.

\begin{tabular}{cccc}
\hline & The Proposed Method & DISPAT & FILTERSIM \\
\hline CPU time(s) & 27,693 & 34,306 & 38,431 \\
Maximum memory $(\mathrm{MB})$ & 289 & 270 & 282 \\
\hline
\end{tabular}

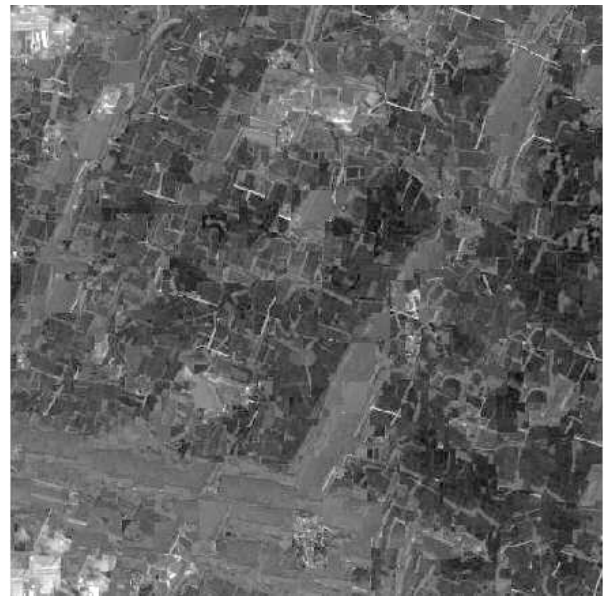

(a)

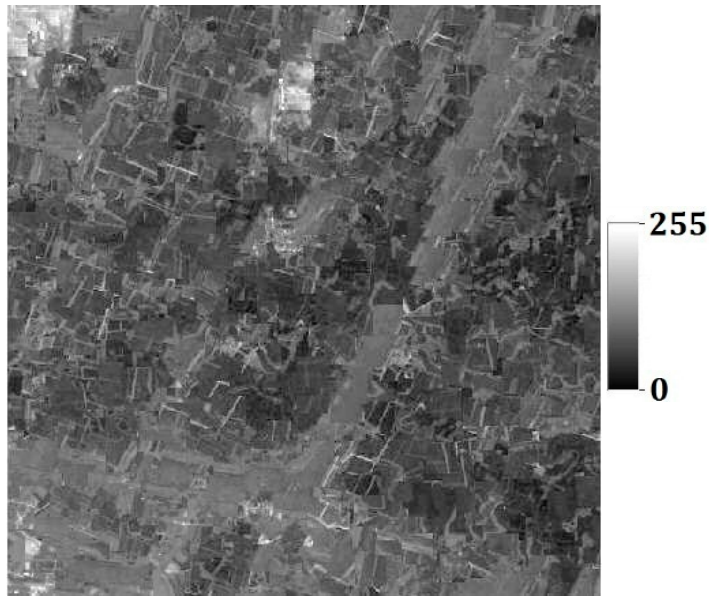

(b)

Figure 29. Super-resolution realizations by FILTERSIM based on the training image of Figure 22b and coarse fraction data of Figure 20b. (a) Case \#4, Realization \#1; (b) Case \#4, Realization \#2.

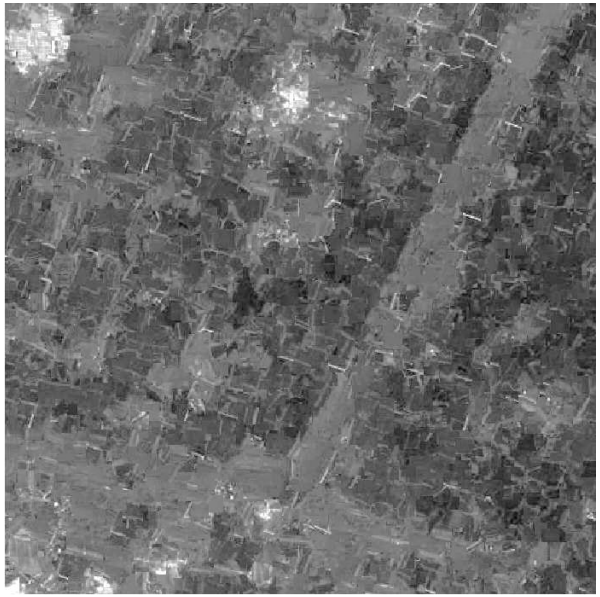

(a)

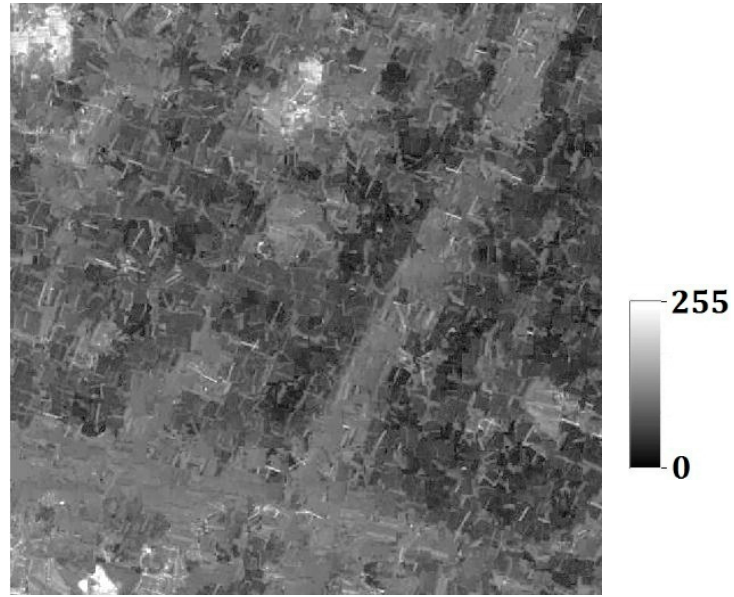

(b)

Figure 30. Super-resolution realizations by DISPAT based on the training image of Figure $22 \mathrm{~b}$ and coarse fraction data of Figure 20b. (a) Case \#4, Realization \#1; (b) Case \#4, Realization \#2. 


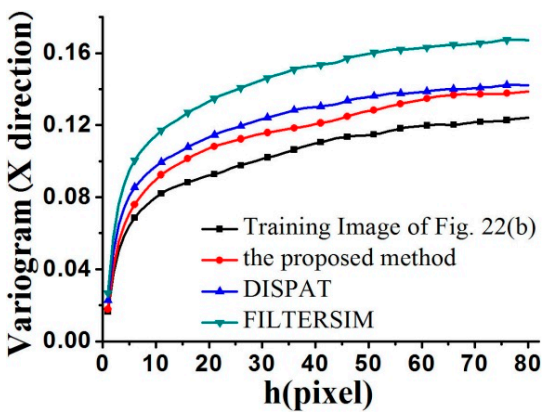

(a)

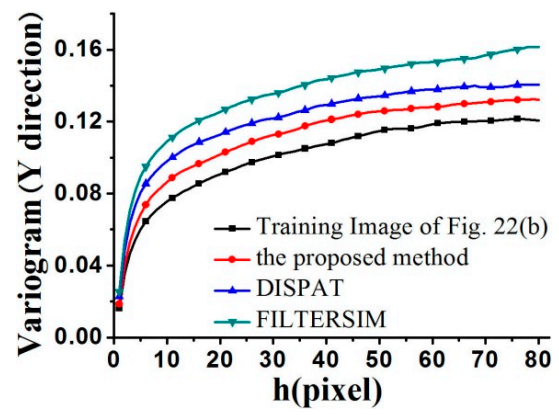

(b)

Figure 31. Standardized variograms of the training image (Figure 22b), standardized average variograms of 100 reconstructions using the proposed method, DISPAT and FILTERSIM in the $X$ and $\mathrm{Y}$ directions (coarse fraction data are from Figure 20b). (a) X direction; (b) Y direction.

\subsection{Discussion for the Results of the Proposed Method, FILTERSIM, and DISPAT}

Theoretically, a higher dimensionality makes classification easier, but uses more time. Recall that the low dimensionality for both FILTERSIM and DISPAT is 6, and that of the proposed method is 4. Hence, it is expected that FILTERSIM and DISPAT use more time, but with better performance in reconstruction quality, which does not accord with the fact that the proposed method has better reconstruction quality and is faster.

The reasons are as follows. First, the data with lower dimensionality helps $k$-means (both used in the above three methods for the classification of patterns) partition the acquired patterns better than those with higher dimensionality. Second, the time complexity of $k$-means is $O\left(N_{p a t} k_{c l s} t\right)$, where $N_{p a t}$ is the number of patterns, $k_{c l s}$ is the number of clusters, and $t$ is the iterative time. Data with lower dimensionality leads to less computational time in clustering, as they are associated with fewer iterations in $k$-means, meaning a smaller $t$ for the proposed method. Finally, ISOMAP has its advantage over the linear dimensionality-reduction methods used in FILTERSIM and DISPAT (i.e., the filters in FILTERSIM and MDS in DISPAT) to achieve better low-dimensional results characterizing the intrinsic features of patterns, leading to better classification.

\section{Conclusions}

Missing or partial spatial data causes the uncertainty and multiple possible results for super-resolution reconstruction of remote sensing images. Super-resolution reconstruction can be viewed as an under-determined problem when reconstructing a fine resolution image from a set of coarse fractions. Prior structural information can be invoked to reduce or constrain the inherent ambiguity in the super-resolution reconstruction. In this paper, the prior information is extracted by collecting the patterns from training images.

MPS is one of the typical methods for stochastic reconstruction, which can extract the intrinsic features in training images, and export them to the simulated regions. Some classical MPS methods, based on linear dimensionality reduction, can hopefully reduce the dimensionality of patterns in high-dimensional space, to shorten simulation time and raise simulation quality, but their congenital linear essence has limited the application for nonlinear data that are widely existent in the real world. Therefore, in our method, ISOMAP (being a classical nonlinear method for dimensionality reduction) is introduced to achieve nonlinear dimensionality reduction, and combined with MPS to address the above issue. A clustering algorithm is performed to classify these patterns after dimensionality reduction. Afterwards, a reconstruction procedure is designed to sequentially reproduce the features of the training images.

In our method, there are in total, two types of simulation, depending on the available conditional data: (1) the simulation conditioned to soft data, (2) the simulation conditioned to both soft data 
and hard data. Coarse fraction information from low-resolution remote sensing images that share the intrinsic characteristics with the training images is viewed as soft data, and always present, but initially there is no available hard data. Further, the whole reconstruction turns into being conditioned to both soft data and hard data, with the increase of simulated pixels. Four case studies demonstrate the validity of our method for the super-resolution reconstruction of remote sensing images, including two binary categorical cases, and two continuous cases. Also, our method has shown its advantage over typical MPS methods (i.e., FILTERSIM and DISPAT) using the linear methods of dimensionality reduction. The tests using both finer-resolution and coarser-resolution coarse fraction data prove the former helps to enhance the reconstruction quality better.

At last, note that reconstructing a super-resolution remote sensing image is often not the ultimate aim, because super-resolution images usually are used as input data, at the fine resolution required by detailed spatial analysis operations and decision support systems, which need to investigate the uncertainty related to the super-resolution remote sensing images. The methodology presented in this paper for generating multiple possible super-resolution remote sensing images, can be used towards such an uncertainty assessment for above operations. Our method can also hopefully be used for general super-resolution image reconstruction to recreate a high-resolution image from low-resolution images, which will be studied in our future work.

Acknowledgments: This work is supported by CAS Strategic Priority Research Program (No. XDB10030402), CNPC-CAS Strategic Cooperation Research Program (No. 2015A-4812), the "Dawn" Program of Shanghai Education Commission (No. 16SG47), the National Natural Science Foundation of China (Nos. 41672114, 61572311) and the Natural Science Foundation of Shanghai (No. 16ZR1413200).

Author Contributions: Ting Zhang proposed the research, organized the entire experimental program, and was involved in the writing of the manuscript. Yi Du was responsible for field data collection and drafted the manuscript. The corresponding author Fangfang Lu drafted part of the manuscript and contributed to the revision of the manuscript.

Conflicts of Interest: The authors declare no conflict of interest.

\section{References}

1. Boucher, A.; Kyriakidis, P.C. Super-resolution land cover mapping with indicator geostatistics. Remote Sens. Environ. 2006, 104, 264-282. [CrossRef]

2. Boucher, A.; Kyriakidis, P.C.; Cronkite-Ratcliff, C. Geostatistical solutions for super-resolution land cover mapping. IEEE Trans. Geosci. Remote Sens. 2008, 46, 272-283. [CrossRef]

3. Wang, G.; Gertner, G.Z.; Anderson, A.B. Spatial-variability-based algorithms for scaling-up spatial data and uncertainties. IEEE Trans. Geosci. Remote Sens. 2004, 42, 2004-2015. [CrossRef]

4. Atkinson, P.M.; Pardo-Igúzquiza, E.; Chica-Olmo, M. Downscaling cokriging for super-resolution mapping of continua in remotely sensed images. IEEE Trans. Geosci. Remote Sens. 2008, 46, 573-580. [CrossRef]

5. Pardo-Igúzquiza, E.; Chica-Olmo, M.; Atkinson, P.M. Downscaling cokriging for image sharpening. Remote Sens. Environ. 2006, 102, 86-98. [CrossRef]

6. Tatem, A.J.; Lewis, H.G.; Atkinson, P.M.; Nixon, M.S. Super-resolution land cover pattern prediction using a Hopfield neural network. Remote Sens. Environ. 2002, 79, 1-14. [CrossRef]

7. Pan, Z.; Yu, J.; Huang, H.; Hu, S.; Zhang, A.; Ma, H.; Sun, W. Super-resolution based on compressive sensing and structural self-similarity for remote sensing images. IEEE Trans. Geosci. Remote Sens. 2013, 51, 4864-4876. [CrossRef]

8. Zhang, K.; Gao, X.; Tao, D.; Li, X. Single image super-resolution with multiscale similarity learning. IEEE Trans. Neural Netw. Learn. Syst. 2013, 24, 1648-1659. [CrossRef] [PubMed]

9. Tang, Y.; Yuan, Y.; Yan, P.; Li, X. Single-image super-resolution via sparse coding regression. In Proceedings of the 18th International Conference on Image Processing, Brussels, Belgium, 11-14 September 2011; pp. 267-272.

10. Kim, K.I.; Kwon, Y. Single-image super-resolution using sparse regression and natural image prior. IEEE Trans. Pattern Anal. Mach. Intell. 2010, 32, 1127-1133. [PubMed] 
11. Li, X.; Orchard, M.T. New edge-directed interpolation. IEEE Trans. Image Process. 2001, 10, $1521-1527$. [PubMed]

12. Li, M.; Nguyen, T. Markov random field model-based edge-directed image interpolation. IEEE Trans. Image Process. 2008, 17, 1121-1128. [PubMed]

13. Huang, C.; Townshend, J.R.G.; Liang, S.; Kalluri, S.N.V.; Defries, R.S. Impact of sensor's point spread function on land cover characterization: Assessment and deconvolution. Remote Sens. Environ. 2002, 80, 203-212. [CrossRef]

14. Kim, S.P.; Su, W.Y. Recursive high-resolution reconstruction of blurred multiframe images. IEEE Trans. Image Process. 1993, 2, 534-539. [CrossRef] [PubMed]

15. Li, X.; Gao, X.; Hu, Y.; Tao, D.; Ning, B. A multi-frame image super-resolution method. Signal Process. 2010, 90, 405-414. [CrossRef]

16. Pradines, D. Improving spot images size and multispectral resolution. In Proceedings of the Earth Remote Sensing Using the Landsat Thermatic Mapper and SPOT Sensor Systems (SPIE), Innsbruck, Austria, 25 November 1986; Voulme 660, pp. 98-102.

17. Freeman, W.T.; Jones, T.R.; Pasztor, E.C. Example based super resolution. IEEE Comput. Graph. Appl. 2002, 22, 56-65. [CrossRef]

18. Chang, H.; Yeung, D.Y.; Xiong, Y. Super-resolution through neighbor embedding. In Proceedings of the IEEE Conference on Computer Vision \& Pattern Recognition, Washington, DC, USA, 27 June-2 July 2004; pp. 275-282.

19. Gao, X.; Zhang, K.; Li, X.; Tao, D. Joint learning for single-image super-resolution via a coupled constraint. IEEE Trans. Image Process. 2012, 21, 469-480. [PubMed]

20. Zhang, H.; Huang, B. Support vector regression-based downscaling for intercalibration of multiresolution satellite images. IEEE Trans. Geosci. Remote Sens. 2013, 51, 1114-1123. [CrossRef]

21. Zhang, K.; Gao, X.; Tao, D.; Li, X. Single image super-resolution with non-Local means and steering kernel regression. IEEE Trans. Image Process. 2012, 21, 4544-4556. [CrossRef] [PubMed]

22. Guardiano, F.; Srivastava, R.M. Multivariate geostatistics: Beyond bivariate moments. In Geostatistics-Troia; Soares, A., Ed.; Kluwier Academic: Dordrecht, The Netherland, 1993; pp. 133-144.

23. Zhang, T.; Switzer, P.; Journel, A. Filter-based classification of training image patterns for spatial simulation. Math. Geosci. 2006, 38, 63-80. [CrossRef]

24. Honarkhah, M.; Caers, J. Stochastic simulation of patterns using distance-based pattern modeling. Math. Geosci. 2010, 42, 487-517. [CrossRef]

25. Choi, H.; Choi, S. Robust kernel Isomap. Pattern Recognit. 2007, 40, 853-862. [CrossRef]

26. Roweis, S.; Saul, L. Nonlinear dimensionality reduction by locally linear embedding. Science 2000, 290, 2323-2326. [CrossRef] [PubMed]

27. Raducanu, B.; Dornaika, F. Embedding new observations via sparse-coding for non-linear manifold learning. Pattern Recognit. 2014, 47, 480-492. [CrossRef]

28. Tenenbaum, B.J.; Silva, V.; Langford, C.J. A global geometric framework for nonlinear dimensionality reduction. Science 2000, 290, 2319-2323. [CrossRef] [PubMed]

29. Strebelle, S.B. Conditional simulation of complex geological structures using multiple-point statistics. Math. Geosci. 2002, 34, 1-21.

30. Hougardy, S. The Floyd-Warshall algorithm on graphs with negative cycles. Inf. Process. Lett. 2010, 110, 279-281. [CrossRef]

31. Levina, E.; Bickel, P.J. Maximum likelihood estimation of intrinsic dimension. In Proceedings of the Neural Information Processing Systems (NIPS 2005), Vancouver, BC, Canada, 5-8 December 2005; pp. 777-784.

32. Vladimir, E.C. Why so many clustering algorithms-A position paper. ACM SIGKDD Explor. Newslett. 2002, 4, 65-75.

33. Peled, S.H.; Mazumdar, S. On coresets for k-means and k-median clustering. In Proceedings of the 36th Annual ACM Symposium on Theory of Computing, Chicago, IL, USA, 13-16 June 2004; pp. 291-300.

34. Goovaerts, P. Geostatistics for Natural Resources Evaluation; Oxford University Press: New York, NY, USA, 1997; pp. 101-122.

(C) 2017 by the authors. Licensee MDPI, Basel, Switzerland. This article is an open access article distributed under the terms and conditions of the Creative Commons Attribution (CC BY) license (http://creativecommons.org/licenses/by/4.0/). 